\title{
Concentration fluctuation in binary polymer blends: $\chi$ parameter, spinodal and Ginzburg criterion
}

\author{
Zhen-Gang Wang \\ Division of Chemistry and Chemical Engineering, California Institute of Technology, \\ Pasadena, California 91125
}

(Received 22 January 2002; accepted 5 April 2002)

\begin{abstract}
A theory for concentration fluctuations in binary polymer blends is developed using field-theoretic techniques. The theory provides a simple, unified framework for addressing a number of important issues. First, consideration of the fluctuation and correlation effects on different length scales leads to a clarification of three different $\chi$ parameters and their interrelationship. By incorporating interaction (modeled by the bare $\chi^{b}$ ) and packing effects up to the polymer size, an effective $\chi^{e}$ emerges as the natural parameter for characterizing the molecular compatibility of the two polymer species. The measured quantity in small-angle neutron scattering (SANS) experiments is an apparent $\chi^{a}$ that includes long wavelength critical and spinodal fluctuations, and is related to $\chi^{e}$ through a self-consistent equation. $\chi^{a}$ exhibits the typical upward parabolic composition dependence observed in experiments and computer simulations. Second, a unified Ginzburg criterion involving both the composition and temperature (or temperaturelike variable) is derived that is applicable to both the critical and the off-critical spinodal regimes. The common characterization of the Ginzburg criterion in terms of a range of temperature (or temperaturelike variable) alone is generally inadequate. The molecular weight scaling proposed by de Gennes and Binder in the respective critical and off-critical spinodal regimes are recovered as special cases in the limit of large molecular weights. For typical molecular weights used in experiments the Ginzburg region is larger than commonly believed. Finally, the nature of the thermodynamic spinodal is examined. It is shown that a true off-critical thermodynamic spinodal does not exist in spatial dimensions less than 4 . In its place, a pseudo-spinodal can be defined where the susceptibility reaches a finite maximum. The pseudo-spinodal precedes the mean-field spinodal but approaches the latter in the limit of infinite molecular weights. The pseudo-spinodal correlates strongly with the free energy barrier for nucleation becoming order $k T$. Thus it provides a kinetic limit for the physically accessible metastable state, beyond which phase separation may exhibit features characteristic of spinodal decomposition. The calculated location of the pseudo-spinodal for two samples used in a recent experiment of Balsara and co-workers agrees with the onset of spinodal-decomposition-like nucleation observed in the experiement. (C) 2002 American Institute of Physics.
\end{abstract}

[DOI: $10.1063 / 1.1481761]$

\section{INTRODUCTION}

Much of our understanding of the thermodynamic - and indeed dynamic - behaviors of binary polymer blends is based on the Flory-Huggins theory, epitomized in the following simple equation for the free energy of mixing per unit volume: ${ }^{1}$

$$
\frac{f_{\text {mix }}(\phi)}{k T}=\frac{\phi}{N_{A} v_{A}} \ln \phi+\frac{1-\phi}{N_{B} v_{B}} \ln (1-\phi)+\chi \phi(1-\phi),
$$

where $N_{\alpha}, v_{\alpha}(\alpha=A, B)$ are respectively the degree of polymerization and the monomer volume of the $\alpha$-species, $\phi$ is the volume fraction of the A-polymer, and the blend is assumed incompressible. $\chi$ is a phenomenological parameter ${ }^{2}$ that accounts for the interaction between the two polymers. From this free energy, the phase diagram can be easily constructed with a critical point located at

$$
\begin{aligned}
& \phi_{c}=\frac{\left(N_{B} v_{B}\right)^{1 / 2}}{\left(N_{A} v_{A}\right)^{1 / 2}+\left(N_{B} v_{B}\right)^{1 / 2},} \\
& \chi_{C}=\frac{\left[\left(N_{A} v_{A}\right)^{1 / 2}+\left(N_{B} v_{B}\right)^{1 / 2}\right]^{2}}{2 N_{A} N_{B} v_{A} v_{B}} .
\end{aligned}
$$

The spinodal curve, the limit of metastability of the onephase state, is given by

$$
\chi_{s}=\frac{1}{2} \frac{\phi N_{A} v_{A}+(1-\phi) N_{B} v_{B}}{\phi(1-\phi) N_{A} v_{A} N_{B} v_{B}} .
$$

In addition to predicting the thermodynamic state of a binary polymer blend, the mean-field phase diagram can be used to infer the kinetic mechanisms of phase separation. ${ }^{3-5}$ Inside the spinodal curve, the binary mixture is unstable with respect to infinitesimal, long wavelength perturbations and phase separation occurs spontaneously through spinodal decomposition. Between the coexistence curve (the binodal) and the spinodal, a one-phase state is metastable, and the formation of a new phase occurs via nucleation. Thus, within 
mean-field theory, the spinodal plays a crucial role in demarcating the two distinct kinetic mechanisms of phase separation.

The Flory-Huggins theory is a mean-field approximation based on the assumption of random mixing. In this article, we consider the effects of concentration fluctuation on the thermodynamics of binary polymer blends and their kinetic implications. Three interrelated issues will be addressed: the validity of the mean-field description; the nature of the thermodynamic spinodal; and the meaning of the Flory-Huggins parameter $\chi$.

The validity of mean-field theory is determined by the so-called Ginzburg criterion-first proposed by Ginzburg and Levanyuk ${ }^{6}$ as a measure of the proximity to the critical point where mean-field theory breaks down because of long wavelength fluctuations. For binary polymer blends, de Gennes $^{7}$ derived a Ginzburg criterion by comparing the magnitude of the concentration fluctuation on the coexistence curve with the width of the miscibility gap. He demonstrated that the non-mean-field region-hereafter referred to as the Ginzburg region-is limited to a small temperature window very close to the critical temperature $T_{c}:\left|1-T_{c} / T\right| \sim 1 / N$; thus mean-field theory is expected to be a valid description of polymer blends over a wide temperature range. This important insight provides the theoretical basis for the application of mean-field [random phase approximation (RPA)] theory to the interpretation of scattering experiments in polymer blends. ${ }^{8}$ Bates et al. ${ }^{9}$ and Hair et al. ${ }^{10}$ extended de Gennes' derivation to include molecular asymmetry between the two polymers in the blends. Belyakov and Kiselev ${ }^{11}$ and Anisimov et al. ${ }^{12}$ proposed an expression that involves critical amplitudes and exponents using the renormalization group approach. Several experiments have been performed to test the Ginzburg criteria for polymer blends near the critical point. ${ }^{9,10,13-15}$ The Ginzburg criterion was also examined extensively by Dudowicz et al. ${ }^{16}$ and Lifschitz et al. ${ }^{17}$ using input from the lattice cluster theory. ${ }^{18}$ Most of these studies show large discrepancies with de Gennes' prediction and with the generalized expressions given in Refs. 9 and 10.

Binder generalized the concept of the Ginzburg criterion to the study of nucleation. ${ }^{5}$ By comparing the magnitude of the concentration fluctuation at a metastable composition $\phi$ with the distance to the spinodal composition $\phi_{s}$, as well as by examining the free energy barrier for nucleation, he determined that, in $d$-dimension, the Ginzburg region is given by $\left(\phi / \phi_{s}-1\right)^{3-d / 2} \sim N^{1-d / 2}\left(1-T / T_{c}\right)^{d / 2-2}$; thus in threedimension, $\left(\phi / \phi_{s}-1\right) \sim N^{-1 / 3}\left(1-T / T_{c}\right)^{-1 / 2}$. We are not aware of experiments specifically designed to test this Ginzburg criterion, although we recently suggested ${ }^{19}$ that the anomalous findings in the nucleation behavior observed by Lefebvre $e t a l .{ }^{20}$ may be related to the onset of the Ginzburg region.

Because the Ginzburg criteria given by de Gennes and Binder were derived using different arguments, their expressions do not allow a crossover from one to the other as we move from the critical regime to near an off-critical spinodal, and neither arguments can be used to derive the Ginzburg criterion above the critical temperature. A unified derivation of the Ginzburg criterion that covers both the critical and off-critical spinodal regimes is still lacking. In addition, it is not clear how large the molecular weights need to be in order to reach the scaling behavior predicted by de Gennes and Binder. Furthermore, these RPA-based Ginzburg criteria leave ambiguity in the interpretation of the parameters that enter the criteria because the critical temperature predicted by the RPA theory is generally quite different from the true critical temperature.

While fluctuation results in significant modification in the thermodynamic behavior of a binary fluid mixture near the critical point, the existence of a critical point is unaffected in three-dimension. The situation is different for the spinodal. There is general recognition that the spinodal is a mean-field concept. Experimental data ${ }^{21,22}$ and computer simulation results ${ }^{23,24}$ do not show a physically accessible singularity. The lack of a sharp spinodal is usually explained in kinetic terms: ${ }^{25,26}$ near the spinodal the free energy barrier for homogeneous nucleation becomes comparable to $k T$, so that the lifetime of the metastable state becomes comparable to the diffusive relaxation time in the system. Thermodynamically, Lebowitz and Penrose ${ }^{27}$ established the conditions under which a metastable state could exist in the gas-liquid transition by relating to the range of interactions in a van der Waals fluid. A renormalization group study by $\mathrm{Saito}^{28}$ using $\epsilon$-expansion (with $\epsilon=6-d$ ) based on a phenomenological Ginzburg-Laudau free energy functional showed that the spinodal fixed-point is unstable in spatial dimensions less than six, suggesting the nonexistence of a true thermodynamic spinodal in three-dimension. For symmetric diblock copolymers, it was first demonstrated by Fredrickson and Helfand, ${ }^{29}$ based on the work of Brazovskii, ${ }^{30}$ and later by others, ${ }^{31,32}$ and is now widely accepted in the block copolymer community, that the large concentration fluctuations near the order-disorder-transition (ODT) destroy the mean-field spinodal. However, the existence of a thermodynamic spinodal in binary polymer blends has not been questioned in the polymer physics literature. In this work we will show that a true, off-critical thermodynamic spinodal does not exist in spatial dimensions less than four. We will explore the thermodynamic and kinetic consequences of this conclusion.

The issue of the Flory-Huggins parameter is inextricably linked to the other fluctuation-related issues discussed above. Originally introduced as a composition independent parameter to account for the enthalpic effect due to mixing, it is now well-known that the $\chi$ parameter includes both enthalpic and entropic effects, with significant composition, ${ }^{33-35}$ pressure ${ }^{36}$ and often non-simple temperature ${ }^{37}$ dependence for most polymers. Clearly, construction of the phase diagram and application of the Ginzburg criteria requires knowing the temperature and composition dependence of the $\chi$ parameter and specifying which $\chi$ parameter is to be used since there are several possible definitions of $\chi$ once fluctuations are included. Beyond this obvious need, it is the task of any statistical mechanical theory of polymer blends to explain a phenomenalogical parameter in terms of more fundamental properties. From both theoretical and experimental points of view, an understanding of how fluctuations on the different length scales contribute to this all-important parameter is highly desirable. 
A large body of theoretical literature exists on the $\chi$-parameter issue. ${ }^{18,38-46}$ Most work focuses on the effects of monomer structure, local liquid structure, and finite compressibility. Statistical mechanical approaches, such as the PRISM theory, ${ }^{41,42}$ the lattice cluster theory, ${ }^{18,43}$ and other lattice approaches, ${ }^{44,45}$ have yielded many important insights into these effects. We emphasize here that the purpose of our work is not to develop a new quantitative theory for the $\chi$ parameter, but rather a simple and clear elucidation of the essential physical effects on the $\chi$ parameter due to concentration fluctuations. While our coarse-grained field-theoretic approach is not best suited to address certain effects arising from the microscopic details of the polymers, it provides a convenient and unified framework for studying both the local (beyond some microscopic cut-off) and long wavelength fluctuations, with the molecular characteristics included in a few easily interpretable parameters. Previous studies using field-theoretical approaches focused either on the local packing effects ${ }^{47}$ or on long wavelength fluctuations. ${ }^{33,48}$ De la Cruz et al. ${ }^{49}$ incorporated fluctuations on both short and long length scales for symmetric blends using a field-theoretic approach similar to ours. But the work did not focus on the $\chi$ parameter issue and instead predicted a shift in the spinodal as a result of using an unrenormalized version of the theory. ${ }^{50}$ A rather comprehensive study of fluctuation effects in symmetric binary polymer blends was conducted by Singh et $a l^{51}$ using a closure condition for the integral equations developed by Yethiraj and Schweizer. ${ }^{52}$ This work included both local and long wavelength fluctuations and took into account the renormalization effects due to long wavelength fluctuations. However, as commented by the authors themselves, any atomic-based closure is prone to qualitatively incorrect description of long wavelength fluctuations, ${ }^{52,53}$ and we believe their theory indeed missed some qualitative effects due to fluctuations near the off-critical spinodal.

In this article, we attempt a systematic examination of concentration fluctuations in binary polymer blends, which simultaneously addresses the $\chi$ parameter issue, the nature of the spinodal and the Ginzburg criterion. Besides the motivations mentioned above, our effort is particularly motivated by recent experimental results of Balsara and co-workers ${ }^{20}$ on the early stages of nucleation in a metastable binary blend. The experiment used a binary blend made of partially deuterated polymethylbutylene $(A)$ and hydrogeneous polyethylbutylene $(B)$, with degrees of polymerization $N_{A}$ $=3357$ and $N_{B}=4260$, respectively. These authors found that the critical length scales for nucleation, instead of diverging as $\chi$ is increased towards the mean-field spinodal, decrease with increasing $\chi$ and seem to show extrapolated divergence in the metastable part of the phase diagram well before the spinodal. A recent self-consistent field calculation by Wood and Wang ${ }^{19}$ estimates that most of their data lie within or near the Ginzburg region as determined from the free energy barrier for homogeneous nucleation. The issues of spinodal and Ginzburg criterion are both clearly relevant here.

We summarize our results through a description of the organization of the article. In the next section, we derive the free energy of mixing for incompressible binary polymer blends by incorporating concentration fluctuations to oneloop order using a field-theoretic formulation. The zerothorder terms yield the Flory-Huggins free energy Eq. (1.1), with the bare $\chi^{b}$ introduced in the model playing the role of the Flory-Huggins parameter. The first-order terms contain corrections due to both enthalpic and entropic effects. A renormalized theory is obtained by considering fluctuation corrections on different length scales. The renormalization procedure leads naturally to the definition of an effective $\chi^{e}$ that incorporates the local molecular effects and an apparent $\chi^{a}$ that includes long wavelength critical and spinodal fluctuations. In Sec. III, we examine the issues mentioned in this Introduction. First, we discuss the meaning of the different $\chi$ 's and their interrelationship. Our theory yields a simple interpretation of the physical content of the different $\chi$ 's and suggests $\chi^{e}$ as the true characterization of molecular miscibility between two polymers. The SANS measured $\chi^{a}$ includes renormalization due to long wavelength critical and spinodal fluctuations and is related to $\chi^{e}$ through a selfconsistent equation. The renormalized $\chi^{a}$ exhibits the characteristic upward parabolic composition dependence observed in experiments and computer simulations. In Sec. III B, we derive a unified Ginzburg criterion (expressed as a Ginzburg number) based on the inverse susceptibility by considering the relative importance of the long wavelength fluctuation correction to the (renormalized) mean-field term. Our result provides a smooth crossover between the critical and off-critical spinodal regimes. The molecular weight scaling proposed by de Gennes and that by Binder are recovered for very large molecular weights. The nature of the spinodal is discussed in Sec. IIIC. We first show that a true thermodynamic spinodal does not exist in three-dimension. We then examine the effects of spatial dimension, demonstrating that the lower critical dimension for the existence of an offcritical thermodynamic spinodal is four and that mean-field behavior prevails in spatial dimensions greater than six, the latter result being consistent with earlier conclusions reached through examination of the nucleation barrier ${ }^{5,26}$ and renormalization group study. ${ }^{28}$ By considering the physical limit of the inverse susceptibility, we identify a pseudo-spinodal where the susceptibility reaches a finite miximum. The relationship between this pseudo-spinodal and the free energy barrier for homogeneous nucleation is established in Sec. IIIC, where we show that at the pseudo-spinodal the free energy barrier becomes of order $k T$. On this basis, we propose that the pseudo-spinodal be taken as the physical or kinetic spinodal separating the physically accessible metastable state from the unstable state. For the two samples studied in the experiments of Ref. 20, we find that the predicted location of the pseudo-spinodal is very close to the onset of anomalous nucleation behavior. This strongly suggests that the findings in that work are manifestations of the kinetic spinodal effects. Finally, in Sec. IIIE, we recast our results for the simplified case of symmetric blends in order to highlight the scaling dependence of various properties on the degree of polymerization. We present a generalized phase diagram and discuss its thermodynamic and kinetic implications. In Sec. IV, we recapitulate the main points of this work and briefly discuss the approximations and assumptions in 
our theory. Several appendices are provided that contain technical details of the calculations omitted in the main body of the article.

\section{FREE ENERGY OF MIXING BEYOND THE FLORY-HUGGINS THEORY}

\section{A. Model}

We consider an incompressible binary $A / B$ polymer blend in a large volume $\mathcal{V}$. We use a coarse-grained model where the polymer chains are represented as continuous threads obeying Gaussian statistics in the absence of interactions. Interactions in the blend are assumed to be of two types: the hard-core short-ranged repulsion between the monomers is modeled by local incompressibility of the binary liquid mixture, and the enthalpic interaction between the $A$ and $B$ polymers is modeled by a local pseudopotential.

Before we proceed to the mathematical expression of our model, we briefly comment on the parametrization of the model. In the theoretical literature, a polymer chain in the melt and blend is usually charaterized by its degree of polymerization $N$, the monomer volume $v$ and the Kuhn length $b$. The monomer is understood as an effective monomer of a Kuhn unit and the degree of polymerization is similiarly interpreted. In relating to the true monomer size, bond length and degree of polymerization, the characteristic ratio $C_{\infty}$ is required. ${ }^{1}$ Such a characterization is inconvenient when comparing theory with experiments.

In this work, we adopt a different parametrization that is free from such an inconvenience. We characterize a polymer by its volume $V_{\alpha}$ (i.e., the partial molar volume of the polymer $\alpha$ divided by the Avogadro number) and its root-meansquare end-to-end distance $R_{\alpha}(\alpha=A, B)$. A flexible polymer in the melt and blend (with neglibible volume change upon mixing) is completely specified by $V_{\alpha}$ and $R_{\alpha}$, both easily determined experimentally.

The primary quantity we seek to obtain is the Helmholtz free energy of mixing per unit volume as a function of the composition. (We shall not distinguish between the Helmholtz free energy and the Gibbs free energy here since the system is assumed incompressible.) For most of our discussions, we are concerned with spatially homogeneous states where the average concentration is uniform. Because the blend is assumed incompressible, the average volume fraction of one of the polymers, say $A$, uniquely characterizes the composition of the system. Henceforth, we use $\phi$ to denote the volume fraction of the $A$ polymers; the volume fraction of the $B$ polymers is simply $1-\phi$. Here and in the rest of the article, $\phi$ denotes the bulk homogeneous composition. When the context is clear, we also use $\phi$ for the spatially varying concentration for notational simplicity; when a distinction needs to be made explicitly, the latter will be denoted by $\phi(\mathbf{r})$.

For studying fluctuation, it is convenient to consider an open system in equilibrium with a large, homogeneous reservoir. The appropriate free energy is the grand potential, defined as

$$
G(\mu, \mathcal{V})=\mathcal{V} g(\mu)=\mathcal{V}[f(\phi)-\mu \phi],
$$

where $f$ is the Helmholtz free energy density of the system and $\mu$ is a chemical potential-like field that is conjugate to the volume fraction $\phi$ defined as

$$
\mu \equiv \frac{\partial f}{\partial \phi} .
$$

Once $g$ is obtained as a function of $\mu$, the Helmholtz free energy density is calculated from Eq. (2.1), with $\phi$ given by

$$
\phi=-\frac{\partial g}{\partial \mu},
$$

and the free energy of mixing is then obtained from

$$
f_{\text {mix }}(\phi)=f(\phi)-[\phi f(0)+(1-\phi) f(1)] .
$$

We calculate $G(\mu, \mathcal{V})$ by using field-theoretical techniques. First, the grand partition function corresponding to $G(\mu, \mathcal{V})$ is transformed into a multi-fold functional integral of field variables through a serious of identity transformation. ${ }^{54}$ The functional integral is then evaluated through a systematic loop expansion. In this work, we perform the expansion to the one-loop order.

We start with the grand partition function for an open, incompressible system of a two-component polymer blend in equilibrium with a reservoir at chemical potential $\mu$ :

$$
\begin{aligned}
& \Xi(\mu, \mathcal{V}) \equiv \exp [-G(\mu, \mathcal{V})] \\
& =\sum_{n_{A}=0}^{\infty} \sum_{n_{B}=0}^{\infty} \frac{1}{n_{A} ! n_{B} !} \frac{1}{\left(V_{A}\right)^{n_{A}}\left(V_{B}\right)^{n_{B}}} \exp \left(\mu n_{A} V_{A}\right) \\
& \times \int D^{n_{A}}\left\{\mathbf{r}_{A}\right\} \int D^{n_{B}}\left\{\mathbf{r}_{B}\right\} \prod_{\mathbf{r}} \delta\left(\hat{\phi}_{A}(\mathbf{r})\right. \\
& \left.+\hat{\phi}_{B}(\mathbf{r})-1\right) \exp \left[-\sum_{i=1}^{n_{A}} h_{i}^{A}-\sum_{j=1}^{n_{B}} h_{j}^{B}\right. \\
& \left.-\chi^{b} \int d \mathbf{r} \hat{\phi}_{A}(\mathbf{r}) \hat{\phi}_{B}(\mathbf{r})\right] \text {. }
\end{aligned}
$$

In this expression, $h_{i}^{\alpha}$ is the single-chain Gaussian Hamiltonian for the $i$ th polymer of species $\alpha$ that accounts for the chain connectivity

$$
h_{i}^{\alpha}=\frac{3}{2 R_{\alpha}^{2}} \int_{0}^{1} d \tau\left(\frac{d \mathbf{r}_{\alpha}(\tau)}{d \tau}\right)^{2}
$$

and $\hat{\phi}_{\alpha}(\mathbf{r})$ is the instantaneous concentration (volume fraction) of $\alpha$ at $\mathbf{r}$ defined as

$$
\hat{\phi}_{\alpha}(\mathbf{r})=V_{\alpha} \sum_{i=1}^{n_{\alpha}} \int_{0}^{1} d \tau \delta\left[\mathbf{r}-\mathbf{r}_{\alpha}(\tau)\right] .
$$

Here $\tau$ is an internal index along the chain contour that runs from 0 at one end to 1 at the other end. The $\delta$-function in Eq. (2.5) enforces the incompressibility and the last term in the exponential represents the "bare" enthalpic interaction between the two polymers. The notation $\int D^{n} \alpha\left\{\mathbf{r}_{\alpha}\right\}$ denotes integration over all chain configurations of polymers of type $\alpha$. The use of the chain volume $V_{\alpha}$ instead of the cube of the thermal de Broglie wavelength as the volume scale in the 
partition function is a matter of convenience; the effect is a composition-independent shift in the chemical potential that has no consequences on the thermodynamics of interest. The unit of energy is chosen to be $k T$.

Before we proceed to the evaluation of the partition function, a few remarks about the coarse-grained continuum model are in order. Clearly, the coarse-grained description of a polymer chain as a Gaussian thread breaks down at length scales approaching the monomer size. Similarly, both the incompressibility condition and the enthalpic pseudo-potential interaction term should be understood to imply a local spatial averaging of the instantaneous monomer densities over some length scale larger than the monomer size. Therefore, the coarse-grained continuum description implicitly involves a microscopic cut-off length $\lambda$, roughly of the order of the Kuhn length. In a lattice model, the lattice spacing serves as the natural cut-off length. In off-lattice molecular models, the microscopic cut-off is contained in the local liquid-structure which in principle can be extracted from the integral equation approaches. In this work, we will take $\lambda$ as an additional parameter in the model without pursuing its detailed molecular origin. From its interpretation as the minimum length scale for the applicability of the coarse-grained model, $\lambda$ is expected to be independent of the composition.

\section{B. Free energy to the one-loop order}

The partition function Eq. (2.5) cannot be calculated exactly because of the interacting nature of the problem (both incompressibility and enthalpic). To make it amenable to sys- tematic approximations, we rewrite the partition function in the form of a multi-fold functional integral through the introduction of collective variables. The details are provided in Appendix A; the result is (aside from some unimportant normalization factor)

$$
\Xi(\mu, \mathcal{V})=\int D \phi \int D W_{A} \int D W_{B} \exp (-K),
$$

where

$$
\begin{aligned}
K= & \chi^{b} \int d \mathbf{r} \phi(1-\phi)-i \int d \mathbf{r}\left[W_{A} \phi+W_{B}(1-\phi)\right] \\
& +I\left(\mu, W_{A}, W_{B}\right) .
\end{aligned}
$$

In the above equations, $i W_{A}(\mathbf{r}), i W_{B}(\mathbf{r})$ can be considered effective external fields for the $A$ and $B$ chains, respectively, and $I\left[\mu, W_{A}(\mathbf{r}), W_{B}(\mathbf{r})\right]$ is the grand "free energy" of noninteracting Gaussian chains in these effective external fields given by Eq. (A4) in Appendix A. Thus, the interacting-chain problem is transformed into a single-chain problem in fluctuating fields; the initial difficulty due to interaction is now shifted to integration over these fluctuating fields.

We evaluate the functional integral by a systematic loop expansion. ${ }^{55}$ To this end, we introduce a smallness parameter (the loop parameter) $a$ for keeping track of the order of the expansion; this parameter will be eventually set to unity. (Alternatively but equivalently, a true smallness parameter can be identified in terms of the molecular weight by nondimensionlizing the variables. ${ }^{54}$ ) Inserting the loop parameter $a$ into Eq. (2.8), we have

$$
\Xi(\mu, \mathcal{V})=\exp \left[-a^{-1} G(\mu, \mathcal{V})\right]=\int D \phi \int D W_{A} \int D W_{B} \exp \left\{-a^{-1} K\left[\mu ; \phi(\mathbf{r}), W_{A}(\mathbf{r}), W_{B}(\mathbf{r})\right]\right\}
$$

$G(\mu, \mathcal{V})$ is evaluated perturbatively as an expansion in the loop parameter $a$. The zeroth-order approximation corresponds simply to the saddle-point value of $K$; this is the self-consistent field approximation. The first-, second- and higher-order terms correspond to the one-loop, two-loop, and higher-loop corrections. The expansion in $G(\mu, \mathcal{V})$ is then converted into an expansion of the Helmholtz free energy using Eqs. (2.1) and (2.3) and the free energy of mixing is obtained from (2.4). In this work, the various quantities will be calculated only to the one-loop order, as this order suffices for addressing the relevant issues. The details of the calculations are provided in Appendix B; the final result for the Helmholtz free energy of mixing is

$$
\begin{aligned}
f_{\text {mix }}= & \frac{\phi}{V_{A}} \ln \phi+\frac{1-\phi}{V_{B}} \ln (1-\phi)+\chi^{b} \phi(1-\phi) \\
& +\Delta f_{\text {pack }}+\Delta f_{\text {int }},
\end{aligned}
$$

where

$$
\begin{aligned}
& \Delta f_{\text {pack }}= \frac{1}{4 \pi^{2}} \int_{0}^{2 \pi / \lambda} k^{2} d k\left[\phi \ln \left(\frac{\phi V_{A} D_{A}+(1-\phi) V_{B} D_{B}}{V_{A} D_{A}}\right)\right. \\
&\left.+(1-\phi) \ln \left(\frac{\phi V_{A} D_{A}+(1-\phi) V_{B} D_{B}}{V_{B} D_{B}}\right)\right], \quad \text { (2.12) } \\
& \Delta f_{\text {int }}=\frac{1}{4 \pi^{2}} \int_{0}^{2 \pi / \lambda} k^{2} d k \ln \left(1-2 \chi^{b} \frac{\phi(1-\phi) V_{A} V_{B} D_{A} D_{B}}{\phi V_{A} D_{A}+(1-\phi) V_{B} D_{B}}\right) .
\end{aligned}
$$

In Eqs. (2.12) and (2.13), $D_{\alpha}$ is the Debye function

$$
D_{\alpha}\left(x_{\alpha}\right)=2 x_{\alpha}^{-2}\left[x_{\alpha}+\exp \left(-x_{\alpha}\right)-1\right]
$$

with $x_{\alpha}=k^{2} R_{\alpha}^{2} / 6 . \lambda$ is the microscopic cut-off length.

The zeroth-order terms in Eq. (2.11) amount to the Flory-Huggins approximation. The correction terms are separated into a packing contribution and an interaction contribution. The former reflects the effects of molecular asymmetry: $\Delta f_{\text {pack }}=0$ if $V_{A}=V_{B}$ and $R_{A}=R_{B}$. The leading contribution to $\Delta f_{\text {pack }}$ is due to conformation asymmetry manifested through the difference in the "packing length" $l_{\alpha}$ 
defined as $l_{\alpha} \equiv V_{\alpha} / R_{\alpha}^{2} \cdot{ }^{56}$ This term was obtained previously by Bates and Fredrickson. ${ }^{47}$ Mathematically, this leading contribution comes from the large- $k$ behavior of the Debye functions. Differences in molecular architecture ${ }^{47}$ and $V_{\alpha}$ or $R_{\alpha}$ give rise to correction terms due to chain ends that are a factor $\lambda / R$ smaller than the leading term. Here, we only explicitly keep the leading term due to conformation asymmetry; the packing correction to the free energy is thus

$$
\Delta f_{\text {pack }}=\frac{2 \pi}{3 \lambda^{3}}\left[\phi \ln \frac{\bar{l}}{l_{A}}+(1-\phi) \ln \frac{\bar{l}}{l_{A}}\right],
$$

where $\bar{l} \equiv \phi l_{A}+(1-\phi) l_{B}$ is the volume fraction-averaged packing length.

The interaction contribution to the free energy correction $\Delta f_{\text {int }}$ reflects the effects due to the enthalpic interaction between the two polymer species (in the bare theory). To evaluate $\Delta f_{\text {int }}$, we note that the logarithm in Eq. (2.13) has a singularity at $k=0$ when $\chi^{b}$ is at its mean-field spinodal $\chi_{s}$ where

$$
\chi_{s}=\frac{1}{2}\left[\frac{1}{\phi V_{A}}+\frac{1}{(1-\phi) V_{B}}\right]=\frac{1}{2} \frac{1}{\phi(1-\phi)} \frac{\bar{V}}{V_{A} V_{B}}
$$

with $\bar{V} \equiv \phi V_{A}+(1-\phi) V_{B}$. The factor multiplying $2 \chi^{b}$ in Eq. (2.13) can be easily recognized as the RPA structure factor $S_{0}(k)$ for a noninteracting binary polymer blend. ${ }^{8} \mathrm{Be}-$ cause the main effects due to long wavelength critical or spinodal fluctuations arise from the small- $k$ behavior, we approximate $S_{0}(k)$ by the Ornstein-Zernike form:

$$
S_{0}(k)=\frac{1}{2 \chi_{s}\left(1+\xi_{0}^{2} k^{2}\right)},
$$

where

$$
\xi_{0}^{2} \equiv \frac{1}{18} \frac{\bar{l}}{l_{A} l_{B}} \frac{V_{A} V_{B}}{\bar{V}}
$$

This approximation yields the correct small- $k$ behavior up to $k^{2}$ (which is all that is required to capture the leading singular behavior near the spinodal) and gives the correct $1 / k^{2}$ behavior for large $k$ with only a difference of $\frac{2}{3}$ in the prefactor. Performing the fairly straightforward integration, and keeping only the leading terms, we obtain

$$
\Delta f_{\mathrm{int}}=-\frac{18 l_{A} l_{B}}{\pi \lambda \bar{l}} \chi^{b} \phi(1-\phi)+\frac{1}{12 \pi \xi_{0}^{3}}\left[1-\left(1-\frac{\chi^{b}}{\chi_{s}}\right)^{3 / 2}\right] .
$$

Thus, to the one-loop order, the free energy of mixing is

$$
\begin{aligned}
f_{\text {mix }}= & \frac{\phi}{V_{A}} \ln \phi+\frac{1-\phi}{V_{B}} \ln (1-\phi)+\chi^{b} \phi(1-\phi) \\
& +\frac{2 \pi}{3 \lambda^{3}}\left[\phi \ln \frac{\bar{l}}{l_{A}}+(1-\phi) \ln \frac{\bar{l}}{l_{B}}\right]-\frac{18 l_{A} l_{B}}{\pi \lambda \bar{l}} \chi^{b} \phi(1-\phi) \\
& +\frac{1}{12 \pi \xi_{0}^{3}}\left[1-\left(1-\frac{\chi^{b}}{\chi_{s}}\right)^{3 / 2}\right] .
\end{aligned}
$$

Following Eq. (2.2), a chemical potential corresponding to the free energy of mixing $\mu_{\text {mix }}=\partial f_{\text {mix }} / \partial \phi$ can be defined

$$
\begin{aligned}
\mu_{\text {mix }}= & \frac{1}{V_{A}}(\ln \phi+1)-\frac{1}{V_{B}}(\ln (1-\phi)+1)+\chi^{b}(1-2 \phi) \\
& +\frac{2 \pi}{3 \lambda^{3}}\left[\ln \frac{l_{B}}{l_{A}}+\frac{l_{A}-l_{B}}{\bar{l}}\right] \\
& -\frac{18 l_{A} l_{B}}{\pi \lambda \bar{l}^{2}}\left((1-\phi)^{2} l_{B}-\phi^{2} l_{A}\right) \chi^{b} \\
& +\frac{1}{8 \pi \xi_{0}^{3}} \frac{2 \chi^{b} V_{A} V_{B}}{\bar{V}^{2}}\left((1-\phi)^{2} V_{B}-\phi^{2} V_{A}\right)\left(1-\frac{\chi^{b}}{\chi_{s}}\right)^{1 / 2} .
\end{aligned}
$$

Finally, the inverse susceptibility (osmotic compressibility) or the inverse structure factor at zero scattering angle, is obtained from the second derivative of the free energy, which yields

$$
\begin{aligned}
\kappa^{-1}= & \frac{1}{V_{A} \phi}+\frac{1}{V_{B}(1-\phi)}-2 \chi^{b}-\frac{2 \pi}{3 \lambda^{3}}\left(\frac{l_{A}-l_{B}}{\bar{l}}\right)^{2}+\frac{36}{\pi} \frac{l_{A}^{2} l_{B}^{2}}{\lambda \bar{l}^{3}} \chi^{b} \\
& -\frac{1}{2 \pi \xi_{0}^{3}} \frac{\chi^{b} V_{A}^{2} V_{B}^{2}}{\bar{V}^{3}}\left(1-\frac{\chi^{b}}{\chi_{s}}\right)^{1 / 2} \\
& \times\left[1+\frac{1}{2} \frac{\chi^{b}}{\bar{V}}\left((1-\phi)^{2} V_{B}-\phi^{2} V_{A}\right)^{2}\left(1-\frac{\chi^{b}}{\chi_{s}}\right)^{-1}\right] .
\end{aligned}
$$

In calculating the chemical potential and the inverse susceptibility, the weak composition dependence in $\xi_{0}$ is ignored, which becomes exact for $V_{A}=V_{B}$ and $R_{A}=R_{B}$.

We note that for symmetric blends, Eq. (2.19) is essentially the same as the expression derived by de la Cruz et $a l .{ }^{49}$ The difference is only in the numerical factors which results from their using a slightly different extrapolation function for the noninteracting structure factor. On the other hand, Eq. (2.19) differs from the corresponding equation in Ref. 33.

\section{Renormalization}

Equation (2.22) forms the starting point for much of our subsequent analysis and discussion. The inverse susceptibility is obtained through a one-loop expansion and is expressed in terms of the bare parameters of the model. In order to relate to physically measurable parameters and to capture the full effect of summing over an infinite subset of one-loop diagrams, ${ }^{55}$ the theory needs to be renormalized. We do so in two steps based on a consideration of the length scales.

The terms nonsingular in $1-\chi^{b} / \chi_{s}$ reflect the packing and interaction effects due to fluctuation and correlation on molecular length scales. The terms containing the singuler $1-\chi^{b} / \chi_{s}$ arise from the long wavelength concentration fluctuations due to proximity to the critical point and spinodal. 
Focusing on the local molecular effects, we see that the fluctuation corrections lead to a finite shift in the spinodal value of $\chi^{b}$ from that given in Eq. (2.16). Rather than defining a new spinodal in terms of $\chi^{b}$, we absorb the shift through the definition of an effective $\chi^{e}$ :

$$
\chi^{e} \equiv \chi^{b}-\frac{18}{\pi} \frac{l_{A}^{2} l_{B}^{2}}{\lambda \bar{l}^{3}} \chi^{e}+\frac{\pi}{3 \lambda^{3}}\left(\frac{l_{A}-l_{B}}{\bar{l}}\right)^{2},
$$

where, in the spirit of renormalization, we have replaced $\chi^{b}$ in the one-loop term by $\chi^{e}$. The above equation can be trivially solved to yield

$$
\chi^{e}=\chi^{b}\left(1+\frac{18}{\pi} \frac{l_{A}^{2} l_{B}^{2}}{\lambda \bar{l}^{3}}\right)^{-1}+\frac{\pi}{3 \lambda^{3}}\left(\frac{l_{A}-l_{B}}{\bar{l}}\right)^{2}
$$

This prescription for renormalization preserves the Flory-Huggins or RPA form of the inverse susceptibility and leaves the spinodal value unchanged from its mean-field value Eq. (2.16). The resulting theory can thus be considered a fluctuation-renormalized mean-field theory, with $\chi^{b}$ replaced by $\chi^{e}$.

We now turn to the effects of long wavelength fluctuations. Using $\chi^{e}$, we write the inverse susceptibility as

$$
\kappa^{-1}=2 \chi_{s}-2 \chi^{e}-\Delta \kappa^{-1}\left(\chi^{e}\right)
$$

where

$$
\Delta \kappa^{-1}=\frac{1}{2 \pi \xi_{0}^{3}} \frac{\chi^{e} V_{A}^{2} V_{B}^{2}}{\bar{V}^{3}}\left(1-\frac{\chi^{e}}{\chi_{s}}\right)^{1 / 2}\left[1+\frac{1}{2} \frac{\chi^{e}}{\bar{V}}\left((1-\phi)^{2} V_{B}-\phi^{2} V_{A}\right)^{2}\left(1-\frac{\chi^{e}}{\chi_{s}}\right)^{-1}\right],
$$

where we have replaced $\chi^{b}$ in $\Delta \kappa^{-1}$ by $\chi^{e}$, which is consistent to the one-loop order.

Because of the long wavelength fluctuation correction, the critical point or spinodal no longer occurs at $\chi^{e}=\chi_{s}$. The true critical point or spinodal is determined by $\kappa^{-1}=0$. We account for this further renormalization effect by definining an apparent $\chi^{a}$ through

$$
\kappa^{-1}=2 \chi_{s}-2 \chi^{a},
$$

so that

$$
\chi^{a}=\chi^{e}+\frac{1}{2} \Delta \kappa^{-1}\left(\chi^{a}\right)
$$

with

$$
\Delta \kappa^{-1}\left(\chi^{a}\right)=\frac{1}{2 \pi \xi_{0}^{3}} \frac{\chi^{a} V_{A}^{2} V_{B}^{2}}{\bar{V}^{3}}\left(1-\frac{\chi^{a}}{\chi_{s}}\right)^{1 / 2}\left[1+\frac{1}{2} \frac{\chi^{a}}{\bar{V}}\left((1-\phi)^{2} V_{B}-\phi^{2} V_{A}\right)^{2}\left(1-\frac{\chi^{a}}{\chi_{s}}\right)^{-1}\right],
$$

where, in the spirit of renormalization, we have replaced $\chi^{e}$ in $\Delta \kappa^{-1}$ by $\chi^{a}$ since it is the latter that determines the true proximity to spinodal. Our choice of the renormalization prescription once again preserves the RPA form of the structure factor and leaves the (nominal) spinodal value unaltered. Making use of the definition for $\xi_{0}$ [Eq. (2.18)] and $\chi_{s}$ [Eq. (2.16)], we can write the self-consistent equation for $\chi_{a}$ as

$$
\chi^{a}=\chi^{e}+\frac{27 \chi^{a}}{\sqrt{2} \pi}\left(\frac{l_{A} l_{B}}{\bar{l}}\right)^{3 / 2} \frac{\left(V_{A} V_{B}\right)^{1 / 2}}{\bar{V}^{3 / 2}}\left(1-\frac{\chi^{a}}{\chi_{s}}\right)^{1 / 2}\left[1+\frac{1}{4} \frac{\chi^{a}}{\chi_{s}} \frac{\left((1-\phi)^{2} V_{B}-\phi^{2} V_{A}\right)^{2}}{\phi(1-\phi) V_{A} V_{B}}\left(1-\frac{\chi^{a}}{\chi_{s}}\right)^{-1}\right] .
$$

It is instructive to write the above equation as a self-consistent equation for the renormalized structure factor at $k=0$ :

$$
S^{-1}(0)=S_{\mathrm{RPA}}^{-1}(0)+\frac{1}{\pi^{2}}\left[\frac{\chi^{a}}{\phi(1-\phi)} \frac{V_{A} V_{B}}{\bar{V}^{2}} \int^{*} k^{2} d k S(k)-\frac{\left(\chi^{a}\right)^{2}}{[\phi(1-\phi)]^{2}} \frac{1}{\bar{V}^{2}}\left((1-\phi)^{2} V_{B}-\phi^{2} V_{A}\right)^{2} \int^{*} k^{2} d k S^{2}(k)\right]
$$

where $S_{\mathrm{RPA}}^{-1}(0)$ and $S^{-1}(0)$ are respectively given by

$$
S_{\mathrm{RPA}}^{-1}(0)=2\left(\chi_{s}-\chi^{e}\right)
$$

and

$$
S^{-1}(0)=2\left(\chi_{s}-\chi^{a}\right) .
$$

In Eq. (2.31), the superscript * indicates that the integral is to be evaluated using dimensional regularization, ${ }^{55}$ since the short wavelength fluctuations have been absorbed into the definition of $\chi^{e}$. By the same token, we only need the long wavelength part of the structure factor $S(k)$ in the integrals, which we approximate with the Ornstein-Zernike form

$$
S(k)=\frac{S(0)}{1+\xi^{2} k^{2}}
$$

with 


$$
\xi=\xi_{0}\left(1-\chi^{a} / \chi_{s}\right)^{-1 / 2} \text {. }
$$

In Eq. (2.31), the first integral represents fluctuation effects near the critical point. The second integral accounts for fluctuations near the off-critical spinodal. In Appendix C, we show that Eq. (2.31) can be mapped to a $\psi^{3}-\psi^{4}$ field theory with vertex functions for $\psi^{3}$ and $\psi^{4}$ that vanish when $\chi^{e}$ $=0$. Thus the RPA structure factor is exact for an incompressible blend with no interactions between the two polymer species. This exact result cannot be captured by fluctuation theories that start with the Flory-Huggins-de Gennes free energy or other phenomenalogical free energies with $\chi$-independent third- and fourth-order vertex functions.

\section{DISCUSSION OF ISSUES}

\section{A. The $\chi$ parameter}

The renormalization procedure discussed in Sec. IIC lends itself naturally to the clarification of the meaning and interpretation of the $\chi$ parameter.

Clearly one must distinguish among three $\chi$ parameters, each reflecting effects on different length scales. The bare $\chi^{b}$ is the "microscopic" interaction parameter introduced in the model. In an incompressible lattice model, this parameter would correspond to the $(1 / 2) z\left(\epsilon_{A A}+\epsilon_{B B}-2 \epsilon_{A B}\right)$ which is the $\chi$ parameter envisioned in the Flory-Huggins theory, ${ }^{1}$ where $z$ is the lattice coordination number and $\epsilon_{\alpha \beta}$ is the nearest neighbor interaction energy between the $\alpha$ and $\beta$ species $(\alpha, \beta=A, B)$. In the free energy resulting from a "bare" mean-field approximation (corresponding to the zero-loop order), such as RPA or self-consistent field theory, $\chi^{b}$ is the Flory-Huggins parameter.

However, even if we ignore long wavelength fluctuations, the local correlation and fluctuation effects due to chain connectivity and interaction modifies the thermodynamics so that $\chi^{e}$, rather than $\chi^{b}$, determines the thermodynamic state (the miscibility) of the blend. The main effects are twofold: first, the (enthalpic) interaction effect is reduced by a factor that depends on the cut-off length, and, second, entropy loss due to packing of conformationally asymmetric polymers makes the two polymer species less miscible; see Eq. (2.24). The first effect is consistent with the Guggenheim $\operatorname{arguments}^{57}$ that each interior unit in a polymer chain is bonded to two neighboring units, so that the effective coordination number is reduced from $z$ to $z-2$; this effect was observed in Monte Carlo simulation using lattice models ${ }^{58}$ and was also captured in the PRISM study by Singh et al. ${ }^{51}$ for symmetric polymer blends. The effect of conformation asymmetry was first elucidated by Bates and Fredrickson ${ }^{47}$ using a field-theoretic approach. The lattice cluster theory of Freed and Dudowicz ${ }^{43}$ predicts both effects as the leading terms in the Flory-Huggins parameter for incompressible binary blends in the long chain limit. A detailed comparison between the result of the lattice cluster theory or of the PRISM theory on one hand, and Eq. (2.24) on the other, can in principle yield an estimate of the cut-off length. However, since our focus here is the qualitative clarification of the various physical effects rather than a quantitative theory for the $\chi$ parameter, we will not make such an effort. Neverthe- less, it is reassuring that our simple coarse-grained model captures both effects in a simple and transparent way at the one-loop order.

The $\chi^{e}$ defined in Eq. (2.24) has the desired properties as the true $\chi$ parameter for characterizing the molecular effects on polymer blend compatibility. This parameter contains both enthalpic and entropic contributions and incorporates fluctuation and correlation effects up to the polymer length scales. By excluding long wavelength critical and spinodal concentration fluctuations, the $\chi^{e}$ so defined is insensitive to the thermodynamic state (i.e., proximity to criticality or spinodal) of the blend and hence is a reflection of the molecular effects on blend compatibility. In the long-chain limit, $\chi^{e}$ is independent of the molecular weight and architecture. The molecular weight dependence of $\chi^{e}$ arises from ends effects and leads to corrections of $O\left(1 / N^{1 / 2}\right)$ or smaller relative to the leading molecular weight independent terms. These additional terms can be included in Eq. (2.24) without modifying the structure of the theory. When ends effects are taken into account, $\chi^{e}$ will generally depend on the molecular architecture and may acquire additional composition dependence. For example, an $A / B$ binary blend and the corresponding $A-B$ diblock copolymer will have $\chi^{e}$ 's with a relative difference of $O(1 / N)$ from each other. ${ }^{59}$ Theoretically speaking, from the monomer design point of view, the preferred Flory-Huggins parameter is $\chi^{e}$ with the ends effects excluded. In real polymer blends, of course, the ends effects are unavoidable.

In the literature, $\chi^{e}$ and $\chi^{b}$ are often confused with each other. This confusion is the basis for the widely but erroneously accepted notion that RPA theory represents a good approximation for very long polymers or far away from the critical point or spinodal. It should become clear from the discussions above that even in the absence of long wavelength fluctuations, RPA or self-consistent field theory, when interpreted literally, is a poor description of polymer blends or block copolymers. For example, RPA or self-consistent field predicts a mean-field spinodal located at

$$
\chi_{s}^{b}=\frac{1}{2} \frac{\bar{V}}{\phi(1-\phi) V_{A} V_{B}},
$$

whereas when local correlation and fluctuation effects are taken into account, the (mean-field) spinodal is shifted to

$$
\chi_{S}^{b}=\frac{1}{2} \frac{\bar{V}}{\phi(1-\phi) V_{A} V_{B}}\left(1+\frac{18}{\pi} \frac{l_{A}^{2} l_{B}^{2}}{\lambda \bar{l}^{3}}\right)-\frac{\pi}{3 \lambda^{3}}\left(\frac{l_{A}-l_{B}}{\bar{l}}\right)^{2} .
$$

However, in terms of $\chi^{e}$, the latter theory still yields

$$
\chi_{s}^{e}=\frac{1}{2} \frac{\bar{V}}{\phi(1-\phi) V_{A} V_{B}} .
$$

Thus the apparent agreement between these two predictions is a result of mistaking $\chi^{e}$ for $\chi^{b}$. All comparisons in the literature between experiments and RPA or self-consistent field calculations have in fact been (awaredly or unawaredly) using a renormalized version of the mean-field theory; this is the reason for the reasonable agreement between experiments and mean-field predictions. Indeed, had a literal RPA 


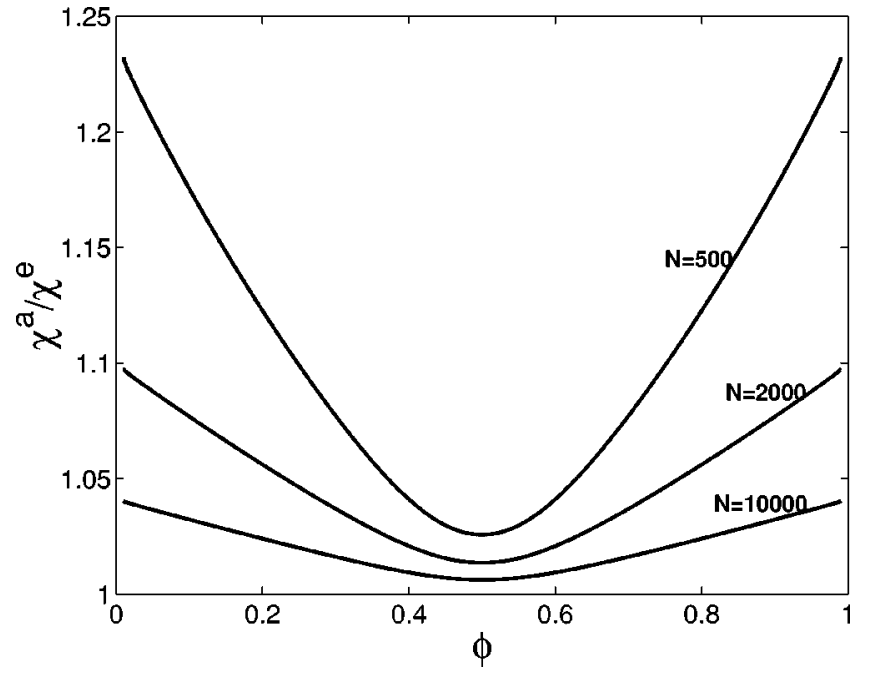

FIG. 1. Composition dependence of the ratio of the apparent $\chi^{a}$ to the true (effective) $\chi^{e}$ for symmetric blends at a fixed $\chi^{e}=0.9 \chi_{c}$. The parameter $b^{3} / v$ is chosen to be $3 . N$ is related to the polymer volume $V$ via $N b^{6} / v^{2}=V / l^{3}$.

or self-consistent field theory been used, predictions for the phase diagram would be far off. When we discuss the validity of mean-field theory in the next section, it is understood that we will be referring to the renormalized mean-field theory that has already taken into account the local correlation and fluctuation effects; in no parts of the phase diagram is the bare mean-field theory valid quantitatively, even for infinitely long polymers.

Although the effective $\chi^{e}$ is the true $\chi$ parameter for characterizing the molecular miscibility of two polymers, its experimental determination is by SANS measurements fitted using the RPA form of the structure factor. The full structure factor inevitably includes long wavelength fluctuations that become important near the critical point or spinodal. Clearly, an RPA fitting yields the apparent $\chi^{a}$, not $\chi^{e} \cdot \chi^{a}$ is determined by $\chi^{e}$ through the self-consisten equation (2.30). The apparent $\chi^{a}$ is always larger than the effective $\chi^{e}$, with a difference that varies inversely with the square-root of the molecular weights. Thus for infinitely long polymers $\chi^{a}$ and $\chi^{e}$ become identical. For polymers with moderate molecular weights, the two can differ from each other visibly, with a larger difference the further away from the critical composition. Figure 1 shows the composition dependence of the ratio of $\chi^{a}$ to $\chi^{e}$ at a fixed $\chi^{e}=0.9 \chi_{c}$ for a symmetric $\left(V_{A}=V_{B}\right.$ $=V, l_{A}=l_{B}=l$ ) blend for three polymer lengths. (The more common characterization in terms of the degree of polymerization $N$, monomer volume $v$ and Kuhn length $b$ is used in the figure; $N b^{6} / v^{2}=V / l^{3}$.) The figure exhibits the upward parabolic shape as observed in experiments ${ }^{33,35}$ and computer simulations. ${ }^{58}$ Similar behavior was also predicted by Singh et al. ${ }^{51}$

The parabolic composition dependence of the measured $\chi$ parameter in isotopic ${ }^{33,60}$ and certain polyolefin ${ }^{35}$ blends has been an unsolved puzzle. The main features that defy a satisfactory explanation are the large magnitude of the increase of the measured $\chi$ at extreme compositions-typically $50 \%$ or larger compared to the value at the critical composition-and seemingly divergent behavior at $\phi=0$ and $\phi=1$ for some isotopic blends. The most recent theoretical work $^{46}$ that examines these unexplained features attributes the observed behavior to experimental errors or to some unknown intrinsic composition dependence in the bare $\chi^{b}$. While long wavelength concentration fluctuations are unable to account for the magnitude of the increase of the experimentally measured $\chi$ at the extreme compositions, the predicted upward increase is not insignificant for moderately long polymers, and the effect can be made more pronounced when density fluctuations are taken into account. ${ }^{51}$ Thus concentration fluctuations could be partially responsible for the experimentally observed behavior. For the parabolic composition dependence observed in computer simulations by Binder and co-workers, ${ }^{58}$ concentration fluctuations (enhanced by coupling with density fluctuations) appear to be a reasonable explanation.

We close this section by a comparison between our fluctuation theory of $\chi$ for binary blends and the corresponding field-theoretically based theory for diblock copolymers. Concentration fluctuations near the order-disorder-transition of diblock copolymers were treated in a seminal work by Fredrickson and Helfand, ${ }^{29}$ using methods developed Brazovskii. ${ }^{30}$ The Fredrickson-Helfand-Brazovskii (FHB) theory provides important insight to the nature of the ODT in block copolymers and forms the theoretical basis for the interpretation of experimental SANS data for diblock copolymers. The theory predicts a significant deviation from the RPA structure in the disordered phase near the ODT which has since been confirmed experimentally. In spite of its many successes, however, the theory has several flaws. First, the theory is essentially phenomenalogical, which used the Leibler mean-field free energy functional in which the order parameter was allowed to fluctuate. While such an approach captures the essential physics in a qualitative and even semiquantitative way, treating fluctuations by allowing the order parameter to fluctuate in a mean-field free energy functional is theoretically unjustified. ${ }^{54}$ This point is shown explicitly for the case of binary polymer blends in Appendix C. Second, the FHB theory is an asymptotic theory where the free energy and its parameters are expanded around the (meanfield) critical point. The theory would predict a finite $\chi^{a}$ even if $\chi^{e}$ is zero. Finally, the Brazovskii method for treating fluctuation used a simple Hartree renormalization where only fluctuation effects due to the quartic nonlinearity are included. The method is incapable of including fluctuation effects due to the cubic term in the free energy that accounts for the asymmetry of the diblock. Just as in the case of blends where renormalization due to the cubic term is almost always as important as or more important than that due to the quartic term, except in the small vicinity of the critical composition (see next section), so we expect that the cubic term in the case of diblock copolymers will have similarly important effects. Thus, strictly speaking, the FHB theory is only applicable to diblock copolymers with a composition very close to the mean-field critical composition (where meanfield theory would predict a direct transition from the disordered phase to the lamellar phase). Recently, Maurer et al. ${ }^{61}$ examined the consistency in using a single $\chi$ parameter to 
characterize the phase behavior for binary blends and the corresponding diblock copolymers, and found discrepancy between the SANS measured $\chi_{A / B}$ for the blend and $\chi_{A-B}$ for the diblock data, with the latter obtained using the FHB theory. Earlier studies by Krishnamoorti ${ }^{62}$ also suggested that the $\chi$ parameter obtained for a binary blend does not always predict the correct phase behavior in the corresponding diblock. Reference 61 attributes this discrepancy to chain stretching of the diblock near the ODT that was not accounted for in the FHB theory, but some or all of the other limitations we have just mentioned may also be responsible.

\section{B. The Ginzburg criterion}

The Ginzburg criterion yields an estimate of the range of temperature in which mean-field theory is valid. From our discussions in Sec. III A, it should be clear that mean-field theory here refers to the renormalized theory in which the local correlation and fluctuation effects have been taken into account. Physically the criterion requires that the fluctuation correction to a physical quantity be much smaller than the quantity itself. The Ginzburg criterion is often expressed in terms of the Ginzburg number $\mathrm{Gi} \equiv\left|1-T_{c} / T_{G}\right|$, so that mean-field theory is valid for $\left|1-T_{c} / T\right| \gg \mathrm{Gi}$. Because of the entropic contribution to the $\chi$ parameter, a Ginzburg number expressed in terms of temperature requires knowledge of the temperature dependence of the $\chi$ parameter. Indeed some of the discrepancies between the Gi obtained in experiments and the theoretical Gi given by de Gennes can be attributed to the complex temperature, composition and pressure dependence in $\chi^{15}$ and do not by themselves necessarily reflect the inadequacy of de Gennes Ginzburg criterion. We avoid this problem by defining the Ginzburg number in terms of the Flory-Huggins parameter $\chi^{e}$ as

$$
\mathrm{Gi} \equiv\left|1-\chi_{G}^{e} / \chi_{s}\right|
$$

Note that we have defined the Ginzburg number with respect to the spinodal value $\chi_{s}$ since we are interested in both the critical region and the region near an off-critical spinodal; the critical point is just a special point on the spinodal at the critical composition. We use $\chi^{e}$ because this quantity is the controlling variable for the thermodynamic behavior of the blend, and we use the mean-field expression $\chi_{s}[$ Eq. (2.16)] because this is the only measurable quantity free from intepretational ambiguity.

In the critical region above $\chi_{c}^{e}$ (or below the critical temperature $T_{c}$ in a UCST system), the Ginzburg criterion is commonly derived by comparing the mean-square composition fluctuation $\left\langle(\delta \phi)^{2}\right\rangle$ with the square of the miscibility gap $\left(\phi^{(1)}-\phi^{(2)}\right)^{2}$, the former quantity being evaluated using the mean-field (RPA) structure factor. In the metastable region, Binder replaced the latter quantity by the square of the difference between the metastable bulk composition and the spinodal composition. As alluded to in the Introduction, this derivation does not provide a crossover between the critical and off-critical spinodal regimes, nor can it be easily extended to the critical region below $\chi_{c}^{e}$ (above $T_{c}$ ). Furthermore, in light of our discussions on the $\chi$ parameter issue, a Ginzburg criterion derived within a mean-field theory leaves an ambiguity as to which of the $\chi$ parameters is to be used, since the critical temperatures determined from using $\chi^{b}$ and $\chi^{e}$ can be far off from each other.

Amit proposed a rational Ginzburg criterion based on comparing the first-order fluctuation correction to the inverse susceptibility to the mean-field susceptibility using renormalized parameters. ${ }^{63}$ Similar strategy was used in the work of Lifschitz et al. ${ }^{17}$ Both studies considered only the critical regime. However, the idea is equally applicable to the offcritical near-spinodal region.

We now derive a unified expression for the Ginzburg number valid both in the critical and off-critical spinodal regimes. To this end, we write the inverse susceptibility as

$$
\kappa^{-1}=2\left(\chi_{s}-\chi^{a}\right)=\kappa_{0}^{-1}-\Delta \kappa^{-1},
$$

where $\kappa_{0}^{-1}$ is the fluctuation-renormalized mean-field part

$$
\kappa_{0}^{-1}=2\left(\chi_{s}-\chi^{e}\right)
$$

and $\Delta \kappa^{-1}$ is the one-loop correction due to long wavelength fluctuations given in Eq. (2.29). The Ginzburg criterion is then expressed as $\Delta \kappa^{-1} \ll \kappa_{0}^{-1}$ or, equivalently, as $\Delta \kappa^{-1}$ $\ll \kappa^{-1}$. Operationally, we define the Ginzburg number by setting $\Delta \kappa^{-1}=c \kappa^{-1}$, i.e., $\kappa_{0}^{-1}=(1+c) \kappa^{-1}$ or

$$
1-\frac{\chi^{e}}{\chi_{s}}=(1+c)\left(1-\frac{\chi^{a}}{\chi_{s}}\right)
$$

where $c$ is a numerical coefficient of order one. The choice of the numerical coefficient is somewhat arbitrary; $c=0.1$ was used in the work of Schwahn and co-workers; ${ }^{14,15,64}$ here we leave it free.

The above equation, together with the self-consistent equation (2.30) determines $\chi_{G}^{e}$, the onset of the Ginzburg region, and the Ginzburg number is then given by Eq. (3.4). The loci of the points determined by Eq. (3.7) are shown in Fig. 4 for a symmetric blend. (Other features of the figure will be discussed in Sec. III E.) Note that the Ginzburg criterion is represented by a curve (hereafter referred to as the Ginzburg curve) in the phase diagram. Thus, specifiying the validity of the mean-field theory requires specifying both $\chi^{e}$ (or $T$ ) and $\phi$. Mean-field theory is valid below this curve. ${ }^{65}$ The usual interpretation of the Ginzburg criterion as a temperature range is inaccurate. The need for an additional variable than the temperature or $\chi$ was suggested earlier by Ref. 16. A few points are worth commenting. First, we note that the minimum of the Ginzburg curve does not occur at the critical composition $\phi_{c}=0.5$. Rather a small maximum appears there. However, overall the portion near the critical composition is fairly flat. Second, part of the binodal curve lies within the Ginzburg region and intersects the Ginzburg curve. The location of the intersection corresponds to a particular $\chi_{G}^{e}{ }^{\prime}$, so that the portion of the binodal below $\chi_{G}^{e}{ }^{\prime}$ is no longer correctly described by mean-field theory. This intersection is the one used by de Gennes in deriving his Ginzburg criterion. ${ }^{7}$

If the Ginzburg number is small, i.e., $\mathrm{Gi} \ll 1$, which corresponds to the case of very long polymers, the Ginzburg number can be shown to satisfiy the following equation: 


$$
\begin{aligned}
\mathrm{Gi}^{1 / 2}= & \frac{27(1+c)^{1 / 2}}{\sqrt{2} c \pi}\left(\frac{l_{A} l_{B}}{\bar{l}}\right)^{3 / 2}\left(\frac{V_{A} V_{B}}{\bar{V}^{2}}\right)^{1 / 2} \bar{V}^{-1 / 2} \\
& \times\left[1+\frac{(1+c)}{4} \frac{\left((1-\phi)^{2} V_{B}-\phi^{2} V_{A}\right)^{2}}{\phi(1-\phi) V_{A} V_{B}} \mathrm{Gi}^{-1}\right] .
\end{aligned}
$$

We now examine several limiting cases of Eq. (3.8).

Near the critical point, Eq. (3.8) simplifies to

$$
\begin{aligned}
\mathrm{Gi}^{1 / 2}= & \frac{27(1+c)^{1 / 2}}{\sqrt{2} c \pi}\left(\frac{l_{A} l_{B}}{\bar{l}}\right)^{3 / 2} \bar{V}^{-1 / 2} \\
& \times\left[1+(1+c)(\delta \bar{\phi})^{2} \mathrm{Gi}^{-1}\right],
\end{aligned}
$$

where $\delta \bar{\phi}$ is the relative deviation from the critical composition defined as

$$
\delta \bar{\phi} \equiv \frac{\phi-\phi_{c}}{\left[\phi_{c}\left(1-\phi_{c}\right)\right]^{1 / 2}}
$$

and where we have made use of the fact that at the critical composition, $V_{A} V_{B} / \bar{V}^{2}=1$ [cf. Eq. (1.2)]. At the critical composition $(\delta \bar{\phi}=0) \chi_{c}=\chi_{s}$. As $\chi^{e}$ approaches the critical $\chi_{c}$ from below, the Ginzburg number is easily solved to be

$$
\mathrm{Gi} \equiv 1-\frac{\chi_{G}^{e}}{\chi_{c}}=\frac{729(1+c)}{2 c^{2} \pi^{2}}\left(\frac{l_{A} l_{B}}{\bar{l}}\right)^{3} \frac{1}{\bar{V}} .
$$

Above $\chi_{c}$, the mean-field composition of the two coexisting phases can be obtained using the renormalized FloryHuggins theory. Near the critical point, the coexistence compositions are given by

$$
\delta \bar{\phi}= \pm \sqrt{3}\left|\chi^{e} / \chi_{c}-1\right|^{1 / 2} .
$$

Substituting this result into Eq. (3.9), we obtain $\chi_{G}^{e}$ ' at the intersection between the mean-field binodal curve and the Ginzburg curve. $\chi_{G}^{e}{ }^{\prime}$ thus defines a special Ginzburg number. This Ginzburg number is usually expressed in terms of the critical (rather than the spinodal) temperature (here the critical $\chi_{c}$ ). On the coexistence curve, it can be shown that

$$
1-\chi^{e} / \chi_{s}=2\left(\chi^{e} / \chi_{c}-1\right)
$$

so that the Ginzburg number in terms of the distance to the critical $\chi_{c}$ becomes

$$
\begin{aligned}
\mathrm{Gi}^{\prime} & \equiv \frac{\chi_{G}^{e} \chi_{c}^{\prime}}{\chi_{c}}-1 \\
& =\frac{729(1+c)}{4 c^{2} \pi^{2}}\left(1+\frac{3}{2}(1+c)\right)^{2}\left(\frac{l_{A} l_{B}}{\bar{l}}\right)^{3} \frac{1}{\bar{V}} .
\end{aligned}
$$

The corresponding range of composition is

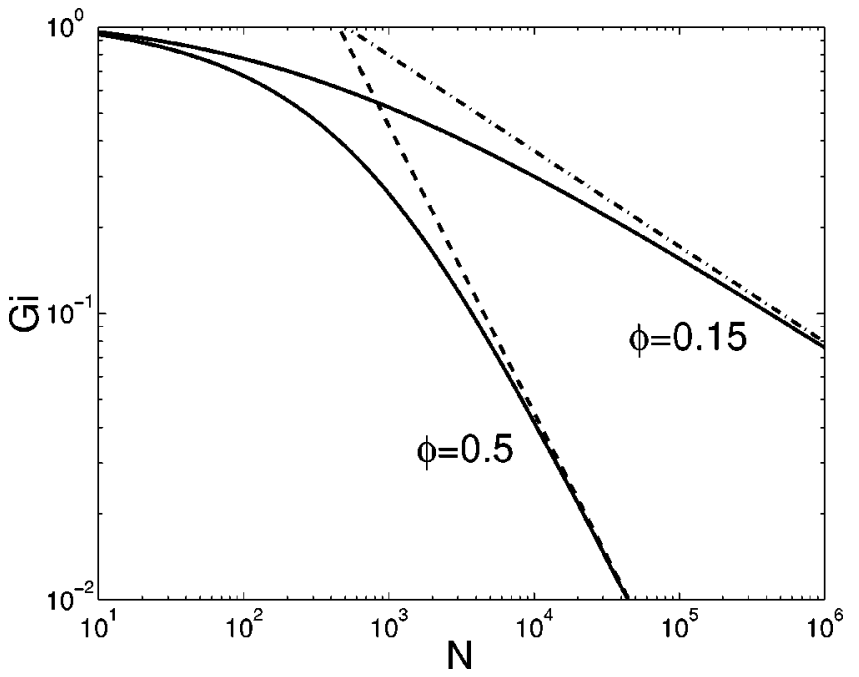

FIG. 2. Dependence of the Ginzburg number on the degree of polymerization for symmetric polymer blends with $b^{3} / v=3$. $c$ is chosen to be 0.1 . The dash and dash-dot lines are the asymptotic scaling behaviors given by Eqs. (3.30) and (3.32), respectively. $N$ is related to the polymer volume $V$ via $N b^{6} / v^{2}=V / l^{3}$.

$$
\begin{aligned}
\left|\phi_{G}-\phi_{c}\right|= & \frac{27}{2 c \pi} \sqrt{\phi_{c}\left(1-\phi_{c}\right)}\left(1+\frac{3}{2}(1+c)\right) \\
& \times\left(\frac{l_{A} l_{B}}{\bar{l}}\right)^{3 / 2} \bar{V}^{-1 / 2} .
\end{aligned}
$$

In terms of the Ginzburg number defined by Eq. (3.4), except very close to the critical composition, the bracket on the rhs of Eq. (3.8) is dominated by the second term. In fact, since $\mathrm{Gi} \rightarrow 0$ in the limit of infinite molecular weights, the second term will always dominate over the first term at any noncritical composition for sufficiently long polymers. Neglecting the subdominant term, we obtain

$$
\begin{aligned}
\mathrm{Gi} \equiv & 1-\frac{\chi_{G}^{e}}{\chi_{s}}=\frac{9}{2(2 c \pi)^{2 / 3}}(1+c)\left(\frac{l_{A} l_{B}}{\bar{l}}\right)\left(\frac{V_{A} V_{B}}{\bar{V}^{2}}\right)^{1 / 3} \\
& \times \bar{V}^{-1 / 3}\left[\frac{\left((1-\phi)^{2} V_{B}-\phi^{2} V_{A}\right)^{2}}{\phi(1-\phi) V_{A} V_{B}}\right]^{2 / 3} .
\end{aligned}
$$

Equations (3.14) and (3.16) recover the limiting scaling expressions near the critical point and near an off-critical spinodal, as first derived by de Gennes and Binder, respectively. However, very large molecular weights are required to reach these scaling results. In Fig. 2, we show the Ginzburg numbers at $\phi=0.5$ and $\phi=0.15$ as a function of the degree of polymerization for symmetric blends. (Recall that for symmetric blends $N b^{6} / v^{2}=V / l^{3}$.) The dash line and the dash-dot line are the analytical predictions given by Eqs. (3.11) and (3.16), respectively [or more directly by Eqs. (3.30) and (3.32)]. For $\phi=0.5, N>10^{4}$ is required to reach the scaling limit. For $\phi=0.15$, we need $N>10^{5}$. Lattice cluster theory calculations in Ref. 17 showed that, in compressible blends, the $1 / N$ scaling for the critical Ginzburg number requires very large $N$. 


\section{The spinodal}

In this section, we discuss the nature of the thermodynamic spinodal for a binary polymer blend.

Thermodynamic spinodal is defined by $\kappa^{-1}=0$, i.e., $\chi^{a}$ $=\chi_{s}$. At the composition such that $(1-\phi)^{2} V_{B}-\phi^{2} V_{A}=0$, the second term in $\Delta \kappa^{-1}$ [Eq. (2.29)] vanishes identically, and the condition $\kappa^{-1}=0$ yields

$$
\chi_{c}^{e}=\chi_{c}^{a}=\frac{1}{2} \frac{\left(V_{A}^{1 / 2}+V_{B}^{1 / 2}\right)^{2}}{V_{A} V_{B}} .
$$

This is just the critical point [cf. Eq. (1.3)].

For $(1-\phi)^{2} V_{B}-\phi^{2} V_{A} \neq 0$, however, the second term in $\Delta \kappa^{-1}$ diverges as $\chi^{a} \rightarrow \chi_{s}$, and no physical solution for $\chi^{e}$ is possible that satisfies $\kappa^{-1}=0$. Thus a true thermodynamic spinodal does not exist! We note that a similar argument was used in demonstrating the disappearance of the spinodal in symmetric diblock copolymer melts. ${ }^{29,30}$

Because fluctuation effects usually depend on spatial dimension, it is of interest to extend our theory to general spatial dimension $d$. The generalization is fairly straightforward with the following result for the inverse structure factor at zero wavenumber:

$$
\begin{aligned}
S^{-1}(0)= & S_{\mathrm{RPA}}^{-1}(0) \\
& +\frac{1}{2} \frac{1}{(2 \pi)^{d}} A_{d}\left[\frac{4 \chi^{a}}{\phi(1-\phi)} \frac{V_{A} V_{B}}{\bar{V}^{2}} \int^{*} k^{d-1} d k S(k)\right. \\
& -\frac{4\left(\chi^{a}\right)^{2}}{[\phi(1-\phi)]^{2}} \frac{1}{\bar{V}^{2}}\left((1-\phi)^{2} V_{B}\right. \\
& \left.\left.-\phi^{2} V_{A}\right)^{2} \int^{*} k^{d-1} d k S^{2}(k)\right]
\end{aligned}
$$

where $A_{d}$ is the area of a $d$-dimension sphere of unit length, $A_{d}=2 \pi^{d / 2} / \Gamma(d / 2)$, with $\Gamma(x)$ the usual gamma-function, and the superscript $*$ again indicates that the integral is to be evaluated using dimensional regularization. In the above equation, the short wavelength fluctuations are understood to have been incorporated through the definition of $\chi^{e}$ using an equation similar to Eq. (2.24). $S_{\mathrm{RPA}}^{-1}$ and $S^{-1}$ are given respectively by Eqs. (2.32) and (2.33), and the OrnsteinZernike form of the structure factor $S(k)$ [Eq. (2.34)] is used in the integrals.

The integrals in Eq. (3.18) can be easily evaluated using standard techniques ${ }^{55}$ to yield

$$
\begin{aligned}
\int{ }^{*} & k^{d-1} d k S^{m}(k) \\
& =S^{m}(0) \xi^{-d} \int \frac{x^{d-1} d x}{\left(1+x^{2}\right)^{m}} \\
& =\frac{1}{2} S^{m}(0) \xi^{-d} \frac{\Gamma(d / 2) \Gamma(m-d / 2)}{\Gamma(m)}, \quad(m=1,2) .
\end{aligned}
$$

Using Eqs. (2.33) and (2.35) for $S(0)$ and $\xi$, and noting that $\chi_{s}$ is given by Eq. (2.16), the above result becomes

$$
\begin{array}{rl}
\int{ }^{*} k^{d-1} & d k S^{m}(k) \\
= & \frac{1}{2} \frac{\Gamma(d / 2) \Gamma(m-d / 2)}{\Gamma(m)} \\
& \times\left(\frac{\phi(1-\phi) V_{A} V_{B}}{\bar{V}}\right)^{m} \xi_{0}^{-d}\left(1-\frac{\chi^{a}}{\chi_{s}}\right)^{-m+d / 2} .
\end{array}
$$

Substituting this result into Eq. (3.18), we obtain

$$
\begin{aligned}
\chi^{a}= & \chi^{e}+\frac{\Gamma(2-d / 2)}{2^{d-1} \pi^{d / 2} \xi_{0}^{d}} \frac{\chi^{a} V_{A}^{2} V_{B}^{2}}{\bar{V}^{3}} \times\left[\frac{1}{d-2}\left(1-\frac{\chi^{a}}{\chi_{s}}\right)^{d / 2-1}\right. \\
& \left.+\frac{1}{4} \frac{\chi^{a}}{\chi_{s}} \frac{\left((1-\phi)^{2} V_{B}-\phi^{2} V_{A}\right)^{2}}{\phi(1-\phi) V_{A} V_{B}}\left(1-\frac{\chi^{a}}{\chi_{s}}\right)^{d / 2-2}\right] .
\end{aligned}
$$

It can be easily checked that the above equation reduces to Eq. (2.30) when $d=3$. As in three-dimension, the first term in the bracket of the above equation is due to critical fluctuation and the second term arises from spinodal fluctuation off the critical composition.

Equation (3.21) allows us to examine the importance of spatial dimension in determining the effects of long wavelength fluctuation. Criticality or the spinodal is defined by $S^{-1}(0)=0$. Clearly, for $\chi^{a}=\chi_{s}$, the first term in the bracket diverges for $d<2$; this is a well-known result indicating that critical point does not exist in spatial dimensions less than two in a binary fluid mixture. ${ }^{66}$ The second term diverges for $d<4$. Therefore, a true off-critical thermodynamic spinodal does not exist in spatial dimensions less than four; long wavelength fluctuations destroy the spinodal predicted by mean-field theory. Thus two and four represent the lower critical dimensions for the existence of the critical point and of the off-critical spinodal, respectively. Further examination of the Ginzburg criteria near the critical point and in the off-critical region near the spinodal shows that the upper critical dimensions (above which mean-field behavior prevails) for the critical point and off-critical spinodal are respectively four and six, in agreement with known results. $5,26,28$ The demonstration is fairly straightforward, so we shall not engage in such an effort. Here we simply summarize the effects of long wavelength fluctuation in different spatial dimensions. For $d<2$, critical point does not exist. For $2<d<4$, critical point exists but near the critical point determined by the Ginzburg criterion, the blend shows nonmean-field behavior; off-critical spinodal does not exist. For $4<d<6$, critical behavior is mean-field-like, off-critical spinodal can exist in the thermodynamic sense (note that this conclusion is different from that suggested by the renormalization group study in Ref. 28), but fluctuation effects become important near the spinodal, which makes it kinetically inaccessible. Constrained Monte Carlo techniques that suppress the unstable fluctuations leading to nucleation ${ }^{67}$ may offer a possible means for observing the thermodynamic spinodal by extending the range of the metastable state beyond the kinetically possible. Finally, for $d>6$, mean-field 
theory becomes an exact description of the full thermodynamic behavior of the blend with a well-defined spinodal.

We now return to the three-dimension world. Because a true spinodal does not exist, the inverse susceptibility cannot reach zero, except at the critical point. To accurately describe the behavior of the susceptibility near the (nominal) spinodal would require going beyond the simple one-loop theory, because well inside the Ginzburg region, higher-loop corrections can no longer be considered small. Nevertheless, some interesting insights can be obtained if we take the one-loop theory as a complete self-consistent theory, which is not entirely groundless, since the one-loop theory is indeed exact for the $O(n)$ model of phase transition in the limit of $n$ $\rightarrow \infty$. ${ }^{55}$

The approach to the (nominal) spinodal is controlled by $\chi^{e}$. However, the proximity of the system to the spinodal is

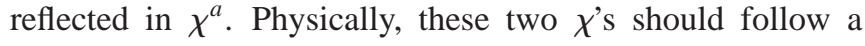
monotonic dependence. The relationship between $\chi^{e}$ and $\chi^{a}$ is shown in Fig. 3. For small $\chi^{e} / \chi_{s}, \chi^{e}$ and $\chi^{a}$ are equal to each other, but begin to deviate from each other noticeably as $\chi^{e} \rightarrow \chi_{s}$. The monotonic increase of $\chi^{a}$ with $\chi^{e}$ terminates at the point shown by the " $\chi$ " in the figure. This point thus signals the physical limit of the one-loop theory, beyond which a stable thermodynamic state is physically impossible. Mathematically, this point corresponds to $\partial \chi^{e} / \partial \chi^{a}=0$. We will term this point the pseudo-spinodal. ${ }^{68}$ An analytical expression can be obtained far off the critical composition for large molecular weights, with the result that

$$
\begin{aligned}
\chi_{p s}^{e}= & \chi_{s}\left\{1-\frac{27}{2(4 \pi)^{2 / 3}}\left(\frac{l_{A} l_{B}}{\bar{l}}\right)\right. \\
& \left.\times\left(\frac{V_{A} V_{B}}{\bar{V}^{2}}\right)^{1 / 3} \bar{V}^{-1 / 3}\left[\frac{\left((1-\phi)^{2} V_{B}-\phi^{2} V_{A}\right)^{2}}{\phi(1-\phi) V_{A} V_{B}}\right]^{2 / 3}\right\} .
\end{aligned}
$$

At the pseudo-spinodal, the correlation length reaches a finite limiting value given by

$$
\xi=\xi_{0}\left(r_{p s}^{a}\right)^{-1 / 2},
$$

where

$$
\begin{aligned}
r_{p s}^{a} \equiv & 1-\frac{\chi_{p s}^{a}}{\chi_{s}}=\frac{9}{2(4 \pi)^{2 / 3}}\left(\frac{l_{A} l_{B}}{\bar{l}}\right)\left(\frac{V_{A} V_{B}}{\bar{V}^{2}}\right)^{1 / 3} \\
& \times \bar{V}^{-1 / 3}\left[\frac{\left((1-\phi)^{2} V_{B}-\phi^{2} V_{A}\right)^{2}}{\phi(1-\phi) V_{A} V_{B}}\right]^{2 / 3} .
\end{aligned}
$$

Since $r_{p s}^{a}$ represents the closest possible distance physically allowed in the one-loop theory, a minimum Ginzburg number $\mathrm{Gi}_{\min }=1-\chi_{p s}^{e} / \chi_{s}$ can be defined for the spinodal, which sets a lower bound for the Ginzburg number given by Eq. (3.16). This lower bound corresponds to $c=2$ or $\Delta \kappa^{-1}$ $=(2 / 3) \kappa_{0}^{-1}$. In the next section, we examine the connection between the pseudo-spinodal and the free energy barrier for nucleation.

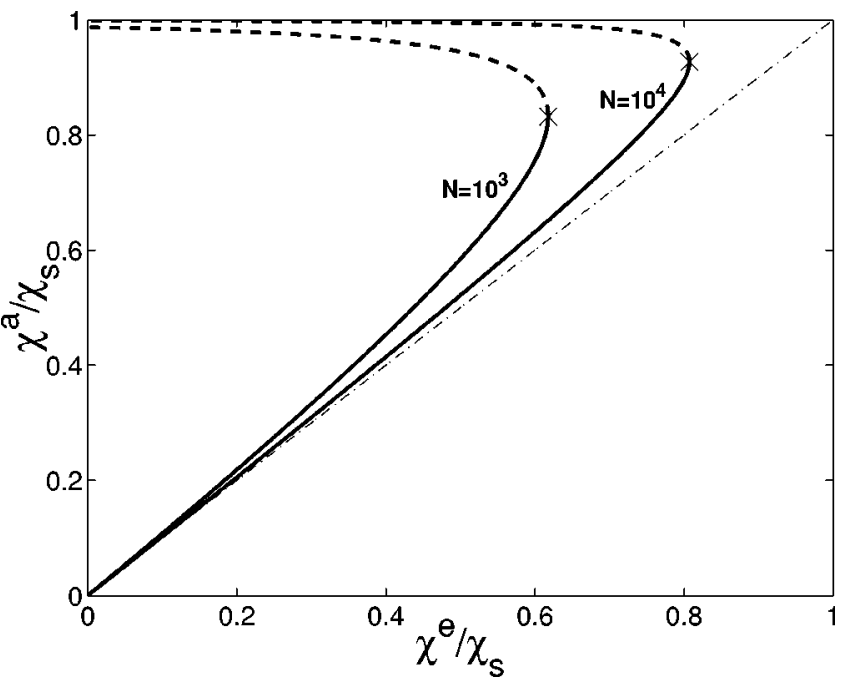

FIG. 3. Relationship between $\chi^{a}$ and $\chi^{e}$ at a fixed composition $\phi=0.1$ for symmetric blends at $N=1000$ and $N=10000$. The parameter $b^{3} / v$ is chosen to be 3. $N$ is related to the polymer volume $V$ via $N b^{6} / v^{2}=V / l^{3}$. The dashed portion of the curve for each case denotes the unphysical solution and the cross $\times$ represents the limit of validity of the theory; this limit is defined as the pseudo-spinodal. The pseudo-spinodal is located at $\chi^{e}$ $=0.618 \chi_{s}, \chi^{a}=0.832 \chi_{s}$ for $N=1000$ and at $\chi^{e}=0.807 \chi_{s}, \chi^{a}=0.927 \chi_{s}$ for $N=10000$

\section{Implications for homogeneous nucleation}

In this section, we explore the relationship between the pseudo-spinodal and the free energy barrier for homogeneous nucleation. An analytical expression for the free energy barrier for a binary fluid mixture close to the spinodal was derived by Cahn and Hilliard ${ }^{69}$ based on a mean-field square-gradient free energy functional. Using a FloryHuggins-de Gennes free energy functional for a weakly inhomogenous binary polymer blend, we can transcribe the Cahn-Hilliard result into an expression in terms of the parameters of the polymer system. This transcription is done in Appendix D; the resulting free energy barrier is

$$
\begin{aligned}
\Delta F_{\text {nucl }} \approx & 2.23\left(\frac{\bar{l}}{l_{A} l_{B}}\right)^{3 / 2}\left(\frac{\bar{V}^{2}}{V_{A} V_{B}}\right)^{1 / 2} \\
& \times \frac{\phi(1-\phi) V_{A} V_{B}}{\left[(1-\phi)^{2} V_{B}-\phi^{2} V_{A}\right]^{2}} \bar{V}^{1 / 2}\left(1-\frac{\chi^{e}}{\chi_{s}}\right)^{3 / 2} .
\end{aligned}
$$

The use of $\chi^{e}$ in the above equation is based on considerations given in Secs. II C and III A.

Binder first suggested the relevance of the Ginzburg criterion to nucleation and indeed used the nucleation free energy barrier as one way to derive the Ginzburg criterion. It can be easily shown that Eq. (3.16) can be obtained by equating this free energy barrier to some multiples of the thermal energy. However, for small $c$ in Eq. (3.16), the Ginzburg criterion would correspond to a large multiple (of order 100 or more) of $k T$ in the nucleation free energy barrier. Thus, conceptually, it is preferrable to distinguish between the onset of the Ginzburg region which simply signals noticeable deviation from mean-field behavior, and the condition of a small nucleation barrier. The pseudo-spinodal, on the other hand, is more strongly correlated with the nucleation free 
energy barrier becoming of order $k T$. At the pseudospinodal, the free energy barrier is estimated to be $8.8 k T$ (recall that $k T$ is our unit of energy in this work). This estimate is close to the $10 k T$ suggested by Binder ${ }^{4,5}$ and used in our recent work. ${ }^{19}$ Since fluctuation at the pseudo-spinodal is quite significant, the actual free energy barrier is likely to be lower.

The condition $\Delta F_{\text {nucl }} \sim k T$ has been suggested by several researchers as defining the intrinsic limit of metastability. 5,26,70,71 The phenomenalogical theories for nucleation rate developed by Langer ${ }^{72}$ and by Patashinskii and Shumilo ${ }^{70}$ indicate that when $\Delta F_{\text {nucl }} \sim k T$, the lifetime of the metastable state becomes comparable to the relaxation time for local equilibrium. (Because of the Ginzburg criterion, the quantitative validity of these theories is questionable very close to the spinodal.) The work of Müller et al. ${ }^{64}$ on homogeneous nucleation and growth in a binary polymer blend in the metastable critical regime reports that the observed nucleation and growth mechanism showed features that resemble spinodal decomposition even for very shallow quenches, suggesting the significant effects of fluctuation on the mechanims of phase separation kinetics.

In light of these considerations, we propose that the pseudo-spinodal defined in Sec. III C be identified as the kinetic spinodal that delineates the boundary between the physically accessible metastable state and the inaccessible (hence presumably unstable) state. We now provide a numerical estimate of the location of the pseudo-spinodal for the experimental systems studied by Lefebvre et al. ${ }^{20}$ Their experiment used a binary blend of partially deuterated polymethylbutylene $(A)$ and hydrogeneous polyethylbutylene (B), with degrees of polymerization $N_{A}=3357$ and $N_{B}$ $=4260$. Two compositions were studied: $\phi=0.161$ (sample B1) and $\phi=0.099$ (sample B2). The work reports unusual behavior in the early stage of nucleation in the ranges 0.84 $<\chi / \chi_{s} \approx 1$ for sample B1 (including one data point at $\chi / \chi_{s}$ $>1$ ) and $0.7<\chi / \chi_{s}<1$ for sample B2. In particular, they found that the critical length scale for nucleation-identified as the inverse of the wavenumber at which the scattering intensity remains unchanged-decreases (rather than increase) as $\chi$ increases towards the mean-field spinodal. For their system, $V_{A}=335700 \AA^{3}, \quad V_{A}=426000 \AA^{3}, \quad l_{A}$ $=2.19 \AA$, and $l_{B}=2.77 \AA$. Using these parameters, the location of the pseudo-spinodal is numerically calculated to be at $\chi^{\prime} \chi_{s}=0.81$ and 0.71 , respectively, for samples B1 and B2. [The approximate equation for the pseudo-spinodal Eq. (3.22) yields 0.81 and 0.69 , repsectively.] These numbers are very close to the corresponding experimental values at the onset of the anomalous nucleation behavior at these two compositions. Based on this agreement, it is reasonable to conjecture that the pseudo-spinodal identified through our one-loop theory provides a good estimate of the kinetic spinodal. Both the behavior of the critical length scale and the time evolution of the structure factor ${ }^{73}$ in the experiments of Lefebvre et al. show features resembling spinodal decomposition. ${ }^{74}$ We thus suggest that the experimental conditions of Ref. 20 were such that the system was already beyond the kinetic spinodal.

\section{E. Simplified results for symmetric blends}

The results in the previous sections are presented for general, conformationally asymmetric blends, because (1) most experimental polymer blends are not molecularly symmetric, and (2) conformation asymmetry leads to important local effects that do not exist in symmetric blends. However, the introduction of additional parameters to characterize an asymmetric blend complicates the appearance of the mathematical results and obscures their simple scaling dependence on the degree of polymerization. In order to highlight this scaling dependence, we consider the special case of symmetric (i.e., $V_{A}=V_{B}$ and $R_{A}=R_{B}$ ) blends, and recast the results using parameters that are more commonly employed in the theoretical literature, namely the degree of polymerization $N$, the Kuhn length $b$ and the monomer volume $v$. We present a generalized phase diagram for the symmetric case and discuss the thermodynamic behavior and the kinetic implications based on this phase diagram.

For a symmetric blend, the entropic contribution due to conformation asymmetry in Eq. (2.24) vanishes, and the relationship between the bare and renormalized $\chi$ assumes the simple form

$$
\chi^{e}=\chi^{b}\left(1+\frac{18}{\pi} \frac{v}{\lambda b^{2}}\right)^{-1} .
$$

We note that, unlike the conformationally asymmetric case where the bare $\chi^{b}$ and the cut-off length $\lambda$ can appear separately, here by expressing $\chi^{b}$ in terms of $\chi^{e}$, it is possible to eliminate the appearance of $\chi^{b}$ and $\lambda$, so that only one parameter, $\chi^{e}$, remains in the renormalized theory.

The self-consistent equation relating the apparent $\chi^{a}$ to the real (effective) $\chi^{e}$, Eq. (2.30), simplifies to

$$
\begin{aligned}
\chi^{a}= & \chi^{e}+\frac{27}{\sqrt{2} \pi} \frac{v}{b^{3}} \chi^{a} N^{-1 / 2}\left(1-\frac{\chi^{a}}{\chi_{s}}\right)^{1 / 2} \\
& \times\left[1+\frac{1}{4} \frac{\chi^{a}}{\chi_{s}} \frac{(1-2 \phi)^{2}}{\phi(1-\phi)}\left(1-\frac{\chi^{a}}{\chi_{s}}\right)^{-1}\right]
\end{aligned}
$$

where now

$$
\chi_{s}=\frac{1}{2} \frac{1}{N v \phi(1-\phi)}
$$

Equation (3.8) for the Ginzburg number in the limit of large $N$ is

$$
\begin{aligned}
\mathrm{Gi}^{1 / 2}= & \frac{27(1+c)^{1 / 2}}{\sqrt{2} c \pi} \frac{v}{b^{3}} N^{-1 / 2} \\
& \times\left[1+\frac{(1+c)}{4} \frac{(1-2 \phi)^{2}}{\phi(1-\phi)} \mathrm{Gi}^{-1}\right] .
\end{aligned}
$$

At the critical composition for $\chi^{e}<\chi_{c}\left(\chi_{c}=2 / N v\right)$, the aymptotic scaling result is

$$
\mathrm{Gi} \equiv 1-\frac{\chi_{G}^{e}}{\chi_{c}}=\frac{729(1+c)}{2 c^{2} \pi^{2}} \frac{v^{2}}{b^{6}} \frac{1}{N} .
$$

and the Ginzburg number $\mathrm{Gi}^{\prime}$ that determines the validity of the binodal curve is 


$$
\mathrm{Gi} \equiv \frac{\chi_{G}^{e \prime}}{\chi_{c}}-1=\frac{729(1+c)}{4 c^{2} \pi^{2}}\left[1+\frac{3}{2}(1+c)\right]^{2} \frac{v^{2}}{b^{6}} \frac{1}{N}
$$

For the off-critical spinodal, the Ginzburg number is

$$
\begin{aligned}
\mathrm{Gi} & \equiv 1-\frac{\chi_{G}^{e}}{\chi_{s}} \\
& =\frac{9}{2(2 c \pi)^{2 / 3}}(1+c) \frac{v^{2 / 3}}{b^{2}} N^{-1 / 3}\left[\frac{(1-2 \phi)^{2}}{\phi(1-\phi)}\right]^{2 / 3} .
\end{aligned}
$$

Finally, the pseudo-spinodal occurs at

$$
\chi_{p s}^{e}=\chi_{s}\left\{1-\frac{27}{2(4 \pi)^{2 / 3}} \frac{v^{2 / 3}}{b^{2}} N^{-1 / 3}\left[\frac{(1-2 \phi)^{2}}{\phi(1-\phi)}\right]^{2 / 3}\right\} \text {. }
$$

We note that the same combination $\bar{N} \equiv N b^{6} v^{-2}$ appears in all the above expressions. This is the same parameter that determines the concentration fluctuation effects in diblock copolymers identified by Fredrickson and Helfand. ${ }^{29}$ Apart from the numerical prefactors, the scaling with $N$ of the Ginzburg number in the critical and off-critical spinodal regimes is identical with the earlier results of de Gennes ${ }^{7}$ and Binder. ${ }^{5}$ However, these scaling results require quite large degrees of polymerization as can be seen from Fig. 2.

The pseudo-spinodal differs from the mean-field spinodal by $\sim N^{-1 / 3}$. In the limit of $N \rightarrow \infty$, the pseudo-spinodal becomes the same as the mean-field spinodal. Thus the thermodynamic spinodal is recovered in the limit of infinite molecular weight. This is consistent with the result reached by renormalization group study of Gunton and Yalabik ${ }^{75}$ for systems with infinitely long-ranged interactions. For moderately long polymers typically used in experiments, because of the slow $N^{-1 / 3}$ decrease and fairly large prefactor (for systems well off the critical composition), the pseudospinodal can occur significantly before the mean-field spinodal. We believe this to be the case for the system studied by Balsara and co-workers.

We summarize the phase behavior for symmetric polymer blends by presenting a generalized phase diagram in Fig. 4. The thick and thin solid lines represent the binodal and mean-field spinodal curves, respectively. Except for possible ends effects, these two curves are independent of $N$. The pseudo-spinodal is indicated by the dash-dot curve $\mathrm{e}^{76}$ and the onset of the Ginzburg criterion (hereafter referred to as the Ginzburg curve) is shown by the dash curve. These two curves depend on $N$ and both move closer to the mean-field spinodal when $N$ increases. The onset of the Ginzburg criterion depends in addition on the choice of $c$; a larger $c$ moves the curve closer to the spinodal. In the figure, we chose $N$ $=3000, b^{3} / v=3$ and $c=0.1$.

We now discuss the thermodynamic and kinetic behaviors based on this phase diagram. Since the phase diagram is symmetric, we focus on the left half. Below the Ginzburg curve (the dash line), mean-field theory provides a good description of the blend. For example, the structure factor is well described by the RPA form (with the effective $\chi^{e}$ ). The Ginzburg curve and the binodal intersect at a special $\chi_{G}^{e}{ }^{\prime}$ with the corresponding $\phi_{G}^{\prime}$. The portion of the binodal for

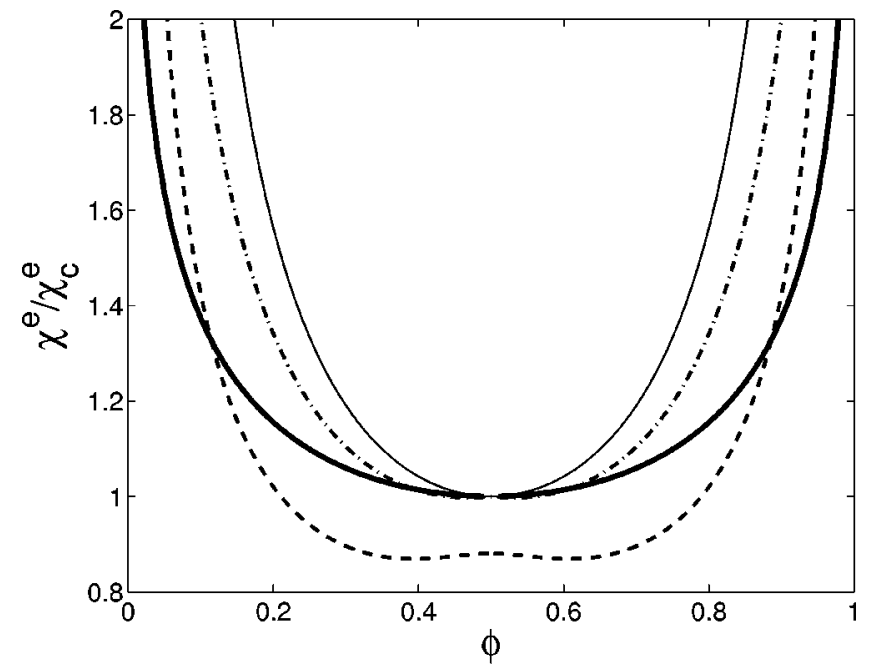

FIG. 4. A generalized phase diagram for a symmetric blend with $N=3000$ and $b^{3} / v=3$ showing the mean-field binodal (thick solid line), the meanfield spinodal (thin solid line), the pseudo-spinodal (dash-dot line), and the loci of the condition (3.7) which defines the Ginzburg criterion (dash line). $N$ is related to the polymer volume $V$ via $N b^{6} / v^{2}=V / l^{3}$. One may notice that the pseudo-spinodal goes slightly below the binodal in the immediate vicinity of the critical point. This artifact is due in part to the use of meanfield theory in calculating the binodal and in part to the breakdown of the one-loop theory near the critical point because of Ising-like fluctuations that can only be captured by renormalization group methods.

$\phi>\phi_{G}^{\prime}$ and $\chi^{e}<\chi_{G}^{e}$ ' will be strongly altered to become Ising-like by large concentration fluctuations. Nucleation in the metastable state of this region will be non-mean-fieldlike and will involve nonclassical exponents. ${ }^{5}$ Above $\chi_{G}^{e}$, between the binodal and the Ginzburg curve, nucleation should be well described by mean-field theory, such as the self-consistent field theory ${ }^{19}$ (with the effective $\chi^{e}$ ). In the metastable region between the Ginzburg curve and the pseudo-spinodal, deviation from mean-field behavior should become significant. However, there can still be a large enough nucleation free energy barrier for the metastable state to be long-lived. When the system reaches the pseudospinodal, the free energy barrier becomes of order $k T$ and the lifetime of the metastable state becomes comparable to the local diffusive relaxation time. The essence of the described scenario was already discussed in the work of Binder. ${ }^{5}$ However, our study represents an improvement in several respects. First, our new phase diagram uses a single unified Ginzburg criterion for both the critical and off-critical spinodal regimes whereas two separate and discontinuous Ginzburg criteria were used in Ref. 5 Second, while the Ginzburg criterion is related to the free energy barrier for nucleation, conceptually we suggest that a distinction be made between the kinetic limit of the metastable state and the Ginzburg criterion which simply signals the onset of significant deviation from RPA-like behavior but does not necessarily imply a small free energy barrier for nucleation. The pseudo-spinodal we introduced in the work is a better measure for the kinetic spinodal. Finally, our general results (allowing conformation asymmetry and applicable for experimentally relevant molecular weights) can be used to make quantitative predictions for specific systems. 


\section{CONCLUSIONS}

The field-theoretic loop expansion provides a systematic technique for studying fluctuation effects in binary polymer blends. We have addressed a number of issues within the framework of a renormalized one-loop theory. By examining the fluctuation effects on different length scales, the physical significance of the various possible definitions of $\chi$ is clarified. It is shown that the real $\chi$ parameter that characterizes the molecular compatibility between two polymers is $\chi^{e}$ which includes fluctuation and correlation effects up to the polymer size arising from both the enthapic interaction (modeled by the bare $\chi^{b}$ ) effects and entropic packing effects due to conformation asymmetry. SANS measurement of the structure factor defines an apparent $\chi^{a}$ which is related to $\chi^{e}$ by a nonlinear self-consistent equation that includes renormalization effects due to long wavelength critical and offcritical spinodal fluctuations. While these qualitative insights are not new, our theory provides a simple and transparent relationship among these different $\chi$ parameters and the selfconsistent equation between $\chi^{a}$ and $\chi^{e}$ includes effects not captured in previous theories.

Our fluctuation theory provides a natural framework for identifying the range of validity of mean-field theory in describing the thermodynamic behavior of a binary polymer blend. By considering the relative importance of the fluctuation correction to the inverse susceptibility, a unified Ginzburg criterion is derived that is applicable to both the critical and the off-critical spinodal regimes and provides a smooth crossover between these two regimes. The complete Ginzburg criterion is shown to always involve the composition, in addition to temperature or $\chi^{e}$. While the Ginzburg number we derived exhibits the same scaling with molecular weight in the respective limiting cases as studied earlier by de Gennes and Binder, very long polymers are required to reach the scaling regime. For moderately long polymers, the nonclassical regions are significantly wider than commonly believed.

An important result of this work concerns the nature of the spinodal. Long wavelength composition fluctuation near the off-critical spinodal produces a stronger singular contribution to the inverse susceptibility than the normal critical fluctuation, resulting in the destruction of the mean-field spinodal in three-dimension. Consequently, even in the purely thermodynamic sense, there is no true divergence in the susceptibility in binary fluid mixtures with a finite spatial range of interactions, except at the critical point. Nevertheless, the distance to the mean-field spinodal when expressed either in terms of the apparent $\chi^{a}$ or the true $\chi^{e}$ (but not the bare $\chi^{b}$ !) provides a meaningful measure of the width of fluctuation-dominant region. By examining the physical limit of the one-loop theory, we identify a pseudo-spinodal at which the correlation length or susceptibility reaches a finite maximum. The pseudo-spinodal precedes the mean-field spinodal by an amount $\sim N^{-1 / 3}$ and merges with the latter in the limit of infinitely long chains. The pseudo-spinodal is shown to be strongly correlated with the free energy barrier for nucleation becoming order $k T$. Therefore we suggest that the pseudo-spinodal be taken as the physical or kinetic spinodal separating the physically accessible metastable state and the unstable state. The calculated location of the pseudospinodal for the two samples used in the recent experimental work of Lefebvre et al. ${ }^{20}$ on nucleation in a binary polymer blend agrees with the onset of the anomalous, spinodaldecomposition-like nucleation behavior found in the experiment, thus strongly suggesting that the system in the experiment was beyond the kinetic spinodal.

We conclude by commenting on the approximations and assumptions in our theory. In this work, we use a coarsegrained model of the polymer blend where the polymers are assumed to be continuous Gaussian chains interacting with a two-body local pseudo-potential subject to a constant local overall density (incompressibility) constraint. In doing so, we have chosen to focus on the effects of concentration fluctuation at length scales larger than the Kuhn lengths of the polymers. Thus we have neglected effects due to local liquid structure and finite compressibility; these effects have been shown to be important contributing factors to the composition, temperature and pressure dependence of the FloryHuggins parameter and have been amply studied by other approaches. These effects generally give rise to a much more involved expression than Eq. (2.24) for the effective $\chi^{e}$ parameter in terms of the molecular parameters. Strong composition dependence in $\chi^{e}$ and the coupling between density and composition fluctuations may add to additional terms to our self-consistent equation (2.30), but the form of the leading singular terms will be unaltered, and most of the qualitative conclusions, such as the nonexistence of a thermodynamic spinodal, will remain valid. It is possible to include the equation of state effects explicitly into the coarse-grained field-theoretic model; ${ }^{77,78}$ in future work we plan to examine such additional effects.

Our one-loop theory represents the lowest-order correction beyond mean-field theory. Renormalization of the parameters in the fluctuation correction terms in effect sums over a subset of an infinite number of terms in the perturbation expansion, thus extending the range of applicability of the theory from its bare form and allowing some important effects (such as the nonexistence of a thermodynamic spinodal) to be captured. However, a loop expansion is inadequate for describing the full effects of long wavelength critical or spinodal fluctuations, such as nonclassical exponents; the renormalization group theory is required. Indeed, Belyakov and Kiselev derived the Ginzburg criterion near the critical point by using the renormalization group method. ${ }^{11}$ However, a renormalization group treatment of the crossover from the critical regime to the off-critical spinodal regime is technically challenging, because these two regimes are governed by two different upper critical dimensions (four and six, respectively). Even for the off-critical spinodal alone, since our physical dimension $d=3$ is far off the upper critical dimension six and is below the lower critical dimension four, the validity of an $\epsilon$-expansion $(\epsilon \equiv 6-d)$ approach is questionable. The one-loop theory, in spite of its limitations, provides a simple, unified theoretical framework for capturing a number of fluctuation effects including the crossover from the critical point to the off-critical spinodal. 


\section{ACKNOWLEDGMENTS}

This work is supported in part by the National Science Foundation (DMR-9970589) and by the China Natural Science Foundation. Helpful discussions with Nitash Balsara, Frank Bates, Karl Freed, Igal Szleifer, and Andrew Spakowitz are gratefully acknowledged.

\section{APPENDIX A: TRANSFORMATION OF THE PARTITION FUNCTION INTO A FUNCTIONAL INTEGRAL}

In this appendix, we briefly sketch the transformation of the partition function Eq. (2.5) into a functional integral. ${ }^{54,79}$

Introducing collective variables $\phi_{\alpha}(\mathbf{r})$ through the identity

$$
\prod_{\mathbf{r}} \int D \phi_{\alpha}(\mathbf{r}) \delta\left(\phi_{\alpha}(\mathbf{r})-\hat{\phi}_{\alpha}(\mathbf{r})\right)=1
$$

and using the Fourier representation of the $\delta$-function, we can rewrite the partition function as a multi-fold functional integral

$$
\begin{aligned}
\Xi(\mu, V)= & \int D \phi_{A} \int D \phi_{B} \int D W_{A} \int D W_{B} \prod_{\mathbf{r}} \delta\left(\phi_{A}(\mathbf{r})\right. \\
& \left.+\phi_{B}(\mathbf{r})-1\right) \exp \left\{-K\left[\phi_{A}(\mathbf{r}), \phi_{B}(\mathbf{r}),\right.\right. \\
& \left.\left.W_{A}(\mathbf{r}), W_{B}(\mathbf{r})\right]\right\},
\end{aligned}
$$

where

$$
\begin{aligned}
K= & \chi \int d \mathbf{r} \phi_{A} \phi_{B}-i \int d \mathbf{r}\left(W_{A} \phi_{A}+W_{B} \phi_{B}\right) \\
& +I\left[\mu, W_{A}, W_{B}\right] .
\end{aligned}
$$

The integration over the auxiliary fields $W_{A}(\mathbf{r})$ and $W_{B}(\mathbf{r})$ results from the Fourier representation of the $\delta$-function in Eq. (A1). In arriving at Eq. (A2), we have replaced $\hat{\phi}_{\alpha}(\mathbf{r})$ by $\phi_{\alpha}(\mathbf{r})$ using the identity Eq. (A1) and have exchanged the order of integration over the functional variables $\phi_{\alpha}(\mathbf{r})$, $W_{\alpha}(\mathbf{r})$ with integration over the configuration of the polymer chains. $I$ is the result of the latter integration and is given by

$$
\begin{aligned}
\exp (-I) & \\
= & \sum_{n_{A}=0}^{\infty} \sum_{n_{B}=0}^{\infty} \frac{1}{n_{A} ! n_{B} !} \\
& \times \frac{1}{\left(V_{A}\right)^{n_{A}}\left(V_{B}\right)^{n_{B}}} \exp \left(\mu n_{A} V_{A}\right) Z_{A}^{n_{A}} Z_{B}^{n_{B}} \\
& \times \exp \left[\frac{\exp \left(\mu V_{A}\right) Z_{A}\left(W_{A}(\mathbf{r})\right)}{V_{A}}+\frac{Z_{B}\left(W_{B}(\mathbf{r})\right)}{V_{B}}\right],
\end{aligned}
$$

where $Z_{A}$ and $Z_{B}$ are the single-chain configurational integrals in the presence of external fields $W_{A}(\mathbf{r})$ and $W_{B}(\mathbf{r})$, respectively. $Z_{\alpha}$ can be obtained from

$$
Z_{\alpha}\left(W_{\alpha}\right)=\int d \mathbf{r} q_{\alpha}(\mathbf{r}, 1)
$$

where $q_{\alpha}(\mathbf{r}, 1)$ is a reduced partition function for a chain with one end at $\mathbf{r}$ and the other end free that satisfies the following diffusion equation

$$
\left(\frac{\partial}{\partial \tau}-\frac{R_{\alpha}^{2}}{6} \nabla_{\mathbf{r}}^{2}+i V_{\alpha} W_{\alpha}(\mathbf{r})\right) q(\mathbf{r} ; \tau)=0
$$

with initial condition $q(\mathbf{r} ; 0)=1$. Because the system is incompressible, the local composition is uniquely specified by either $\phi_{A}(\mathbf{r})$ or $\phi_{B}(\mathbf{r})=1-\phi_{A}(\mathbf{r})$. We denote $\phi_{A}(\mathbf{r})$ simply by $\phi(\mathbf{r})$; the integration over $\phi_{B}(\mathbf{r})$ in Eq. (A2) can be trivially performed to yield Eq. (2.8).

\section{APPENDIX B: FREE ENERGY CALCULATION THROUGH A LOOP EXPANSION}

We start with the representation of the grand partition function Eq. (2.10) and develop an expansion in the loop parameter $a$ to first order in $a$.

The zeroth-order approximation for evaluating the functional integral is the steepest descent or saddle point approximation. This involves finding the stationary point of the functional $K$ with respect to its variables and equating the grand free energy $G$ with the stationary value of $K$. Setting the functional derivatives of $K$ with respect to the field variables to zero and noting that the saddle-point values of $W_{\alpha}$ lie on the imaginary axis, we obtain

$$
\begin{aligned}
& w_{A, 0}(\mathbf{r})-w_{B, 0}(\mathbf{r})=\chi^{b}\left(1-2 \phi_{0}(\mathbf{r})\right), \\
& \phi_{0}(\mathbf{r})=\exp \left(\mu V_{A}\right) \int_{0}^{1} d \tau q_{A}(\mathbf{r}, 1-\tau) q_{A}(\mathbf{r}, \tau),
\end{aligned}
$$

$$
1-\phi_{0}(\mathbf{r})=\int_{0}^{1} d \tau q_{B}(\mathbf{r}, 1-\tau) q_{B}(\mathbf{r}, \tau),
$$

where we have defined $w_{\alpha, 0}=i W_{\alpha, 0}$ so that all the variables are now real and the $q_{\alpha}(\mathbf{r}, 1)$ 's are solved from Eq. (A6). The subscript 0 refers to the saddle-point or zeroth-order approximation.

Equations (B1)-(B3) are nothing but the self-consistent field equations for an inhomogeneous binary polymer blend. They can be easily solved for the case of a homogenous state with uniform composition, with the results

$$
\begin{aligned}
& w_{A, 0}-w_{B, 0}=\chi^{b}\left(1-2 \phi_{0}\right), \\
& \phi_{0}=\exp \left(\mu V_{A}-w_{A, 0} V_{A}\right), \\
& 1-\phi_{0}=\exp \left(-w_{B, 0} V_{B}\right),
\end{aligned}
$$

It is more convenient to write the solutions in terms of $w_{A, 0}, w_{B, 0}$ and $\mu$ as functions of the composition $\phi_{0}$; doing so we obtain

$$
\begin{aligned}
& w_{A, 0}=\chi^{b}\left(1-\phi_{0}\right)-\frac{1}{V_{B}} \ln \left(1-\phi_{0}\right), \\
& w_{B, 0}=-\frac{1}{V_{B}} \ln \left(1-\phi_{0}\right),
\end{aligned}
$$

and

$$
\mu=\frac{1}{V_{A}} \ln \phi_{0}-\frac{1}{V_{B}} \ln \left(1-\phi_{0}\right)+\chi^{b}\left(1-2 \phi_{0}\right) .
$$


These results can be substituted into Eq. (A3) to yield the grand potential per unit volume as

$$
g_{0}=\frac{1}{V_{A}} \ln \left(1-\phi_{0}\right)-\frac{\phi_{0}}{V_{A}}-\frac{1-\phi_{0}}{V_{B}}+\chi^{b} \phi_{0}^{2},
$$

from which we obtain the Helmholtz free energy density

$$
\begin{aligned}
f_{0}\left(\phi_{0}\right)= & \frac{\phi_{0}}{V_{A}}\left[\ln \phi_{0}-1\right]+\frac{1-\phi_{0}}{V_{B}}\left[\ln \left(1-\phi_{0}\right)-1\right] \\
& +\chi^{b} \phi_{0}\left(1-\phi_{0}\right) .
\end{aligned}
$$

The free energy of mixing can be easily obtained with the result

$$
\begin{aligned}
f_{\text {mix }, 0}\left(\phi_{0}\right)= & \frac{\phi_{0}}{V_{A}} \ln \phi_{0}+\frac{1-\phi_{0}}{V_{B}} \ln \left(1-\phi_{0}\right) \\
& +\chi^{b} \phi_{0}\left(1-\phi_{0}\right) .
\end{aligned}
$$

This is just the Flory-Huggins free energy.

To calculate corrections to the mean-field (selfconsistent field) result, we expand the field variables around their saddle-point values:

$$
\begin{aligned}
& \phi(\mathbf{r})=\phi_{0}+\delta \phi(\mathbf{r}), \\
& i W_{A}(\mathbf{r})=w_{A, 0}+i \delta W_{A}(\mathbf{r}), \\
& i W_{B}(\mathbf{r})=w_{B, 0}+i \delta W_{B}(\mathbf{r}) .
\end{aligned}
$$

To order $a$, only the quadratic terms in $\delta \phi(\mathbf{r})$ and $\delta W_{\alpha}(\mathbf{r})$ are required; higher order terms contribute to order $a^{2}$ or higher. ${ }^{55}$ Performing the straightforward Gaussian integrals, we obtain, to order $a$, the grand free energy

$$
\begin{aligned}
G(\mu, \mathcal{V}) & =\mathcal{V} g(\mu) \\
& =\mathcal{V}\left[g_{0}\left(\phi_{0}(\mu)\right)+a g_{1}\left(\phi_{0}(\mu)\right)+O\left(a^{2}\right)\right],
\end{aligned}
$$

where

$$
\begin{aligned}
g_{1}\left(\phi_{0}\right)= & \frac{1}{2(2 \pi)^{3}} \int d \mathbf{k}\left\{\operatorname { l n } \left[\phi_{0} V_{A} D_{A}\left(x_{A}\right)\right.\right. \\
& \left.+\left(1-\phi_{0}\right) V_{B} D_{B}\left(x_{B}\right)\right]+\ln \left[1-2 \chi^{b}\right. \\
& \left.\left.\times \frac{\phi_{0}\left(1-\phi_{0}\right) V_{A} V_{B} D_{A}\left(x_{A}\right) D_{B}\left(x_{B}\right)}{\phi_{0} V_{A} D_{A}\left(x_{A}\right)+\left(1-\phi_{0}\right) V_{B} D_{B}\left(x_{B}\right)}\right]\right\},
\end{aligned}
$$

where $D_{\alpha}\left(x_{\alpha}\right)$ is the Debye function

$$
D_{\alpha}\left(x_{\alpha}\right)=2 x_{\alpha}^{-2}\left[x_{\alpha}+\exp \left(-x_{\alpha}\right)-1\right]
$$

with $x_{\alpha}=k^{2} R_{\alpha}^{2} / 6$. In writing Eq. (B17) we have made use of the saddle-point solution, Eqs. (B7)-(B9).

To order $a$, the volume fraction $\phi$ is obtained by

$$
\phi=-\frac{\partial g}{\partial \mu}=\phi_{0}+a \phi_{1}+O\left(a^{2}\right) .
$$

Now the Helmholtz free energy is obtained by an inverse Legendre transform: $f(\phi)=g(\mu)+\mu \phi$

$$
\begin{aligned}
& =g_{0}\left(\phi_{0}(\mu)\right)+a g_{1}\left(\phi_{0}(\mu)\right)+O\left(a^{2}\right)+\mu \phi \\
& =g_{0}(\phi)+\mu \phi+g_{0}\left(\phi_{0}\right)-g_{0}(\phi)+a g_{1}\left(\phi_{0}\right)+O\left(a^{2}\right) .
\end{aligned}
$$

Due to the stationarity of the $g_{0}$ at $\phi_{0}$, the difference $g_{0}\left(\phi_{0}\right)-g_{0}(\phi)$ is $O\left(a^{2}\right)$. Also, to order $a$, we can replace the argument $\phi_{0}$ by $\phi$ in $g_{1}$. Therefore,

$$
f(\phi)=f_{0}(\phi)+a g_{1}(\phi)+O\left(a^{2}\right),
$$

where $f_{0}(\phi)$ is the same function as Eq. (B11) with $\phi_{0}$ replaced by $\phi$. Equation (2.11) follows from the definition of the free energy of mixing Eq. (2.4).

\section{APPENDIX C: RELATIONSHIP TO PHENOMENALOGICAL FIELD THEORY}

In this appendix, we (1) show that the self-consistent equation for the inverse structure factor Eq. (3.18) corresponds to a one-loop expansion of a $\psi^{3}-\psi^{4}$ field theory, (2) comment on the validity of using the Flory-Huggins-de Gennes free energy for study fluctuation in polymer blends, and (3) conjecture the possible structure of a one-loop fluctuation theory for diblock copolymers.

Consider the following Lagrangian functional:

$$
\begin{aligned}
L= & \int d \mathbf{r}\left\{\frac{1}{2}\left[\left(2 \chi_{s}-2 \chi^{e}\right) \psi^{2}(\mathbf{r})+2 \chi_{s} \xi_{0}^{2}(\nabla \psi(\mathbf{r}))^{2}\right]\right. \\
& \left.+\frac{1}{3 !} \gamma_{3,0} \psi^{3}(\mathbf{r})+\frac{1}{4 !} \gamma_{4,0} \psi^{4}(\mathbf{r})\right\} .
\end{aligned}
$$

Obviously, the RPA structure factor is simply

$$
S_{\mathrm{RPA}}(k)=\frac{1}{2 \chi_{s}-2 \chi^{e}+2 \chi_{s} \xi_{0}^{2} k^{2}} .
$$

Including fluctuations due to the $\psi^{3}$ and $\psi^{4}$ nonlinearity to one-loop order, we obtain

$$
\begin{aligned}
S^{-1}(0)= & S_{\mathrm{RPA}}^{-1}(0)+\frac{1}{2} \frac{A_{d}}{(2 \pi)^{d}} \gamma_{4,0} \int k^{d-1} d k S_{\mathrm{RPA}}(k) \\
& -\frac{1}{2} \frac{A_{d}}{(2 \pi)^{d}}\left(\gamma_{3,0}\right)^{2} \int k^{d-1} d k S_{\mathrm{RPA}}^{2}(k) .
\end{aligned}
$$

Replacing $S_{\mathrm{RPA}}(k)$ by $S(k)$ in the fluctuation correction terms by substituting $\chi^{a}$ for $\chi^{e}$, and similarly in $\gamma_{3,0}$ and $\gamma_{4,0}$, we obtain a renormalized self-consistent equation for $S(0)$ :

$$
\begin{aligned}
S^{-1}(0)= & S_{\mathrm{RPA}}^{-1}(0)+\frac{1}{2} \frac{A_{d}}{(2 \pi)^{d}} \gamma_{4} \int k^{d-1} d k S(k) \\
& -\frac{1}{2} \frac{A_{d}}{(2 \pi)^{d}} \gamma_{3}^{2} \int k^{d-1} d k S^{2}(k),
\end{aligned}
$$

where $\gamma_{3}$ and $\gamma_{4}$ are the same functions as the corresponding $\gamma_{3,0}$ and $\gamma_{4,0}$ with $\chi^{e}$ replaced by $\chi^{a}$.

Comparing the above equation with Eq. (3.18) and recalling the substitution of $\chi^{e}$ by $\chi^{a}$ in the renormalized equation, we identify 


$$
\gamma_{3,0}=\frac{2 \chi^{e}}{\phi(1-\phi)} \frac{1}{\bar{V}}\left[(1-\phi)^{2} V_{B}-\phi^{2} V_{A}\right]
$$

and

$$
\gamma_{4,0}=\frac{4 \chi^{e}}{\phi(1-\phi)} \frac{V_{A} V_{B}}{\bar{V}^{2}} .
$$

We note that the functional Eq. (C1) with the above vertex functions cannot be obtained from an expansion using the Flory-Huggins de Gennes theory. Therefore, previous work that treats fluctuation in binary polymer blends using the Flory-Huggins-de Gennes free energy is not justified from first principles and should be considered phenomenalogical.

For the case of diblock copolymer, an equation similar to Eq. (C4) is expected. In general the last term is nonvanishing except for the symmetric case (where mean-field theory predicts a direct transition from the disordered phase to the lamellar phase) and will contribute significantly to the renormalization equation between $\chi^{a}$ and $\chi^{e}$ near the ODT. Indeed, by simple power counting, one can show that the $\gamma_{3}$ term results in a stronger singularity $\left(1-\chi^{a} / \chi_{s}\right)^{-3 / 2}$ than the $\left(1-\chi^{a} / \chi_{s}\right)^{-1 / 2}$ term due to $\gamma_{4}$ included in the work of Fredrickson and Helfand. ${ }^{29}$ At the phenomenalogical level, the effects due to nonvanishing $\gamma_{3}$ can be addressed starting with the Leibler free energy, a task that remains to be done. However, it should be clear from the discussions in this appendix that the Leibler free energy (the analog of the FloryHuggins-de Gennes free energy) would not yield the correct vertex functions for studying fluctuations.

\section{APPENDIX D: NUCLEATION BARRIER NEAR THE MEAN-FIELD SPINODAL}

In this appendix, we provide an expression for the free energy barrier for homogeneous nucleation near the spinodal. A general theory for nucleation in binary fluid mixture near the spinodal was developed some time ago by Cahn and Hilliard ${ }^{69}$ based on a square-gradient density functional formulation. Near the spinodal, the excess free energy for an inhomogenous system can be expanded as a functional Taylor expansion around the uniform density $\phi$ of the homogeneous metastable state, as

$$
\Delta F=\int d \mathbf{r}\left[\frac{1}{2} f^{(2)} \psi^{2}(\mathbf{r})+\frac{1}{3 !} f^{(3)} \psi^{3}(\mathbf{r})+\zeta\left(\nabla \psi^{2}(\mathbf{r})\right)^{2}\right],
$$

where $\psi(\mathbf{r})=\phi(\mathbf{r})-\phi . f^{(2)}$ and $f^{(3)}$ are respectively the second and third derivatives of the free energy and it should be understood that these derivatives are evaluated at the bulk composition. The absence of the linear term reflects the fact that a metastable state corresponds to a local free energy minimum. Following Cahn and Hilliard, we rewrite the above equation as

$$
\Delta F=\int d \mathbf{r}\left[\eta\left(3 \tau \psi^{2}(\mathbf{r})-\psi^{3}(\mathbf{r})\right)+\zeta(\nabla \psi(\mathbf{r}))^{2}\right],
$$

where

$$
\eta=-\frac{1}{3 !} f^{(3)}
$$

and

$$
\tau=-\frac{f^{(2)}}{f^{(3)}} .
$$

By identifying an inhomogenous saddle point of the above free energy, Cahn and Hilliard obtained the following free energy barrier for the formation of the critical nucleus:

$$
\Delta F_{\text {nucl }} \approx 197 \eta^{-1 / 2} \zeta^{3 / 2} \tau^{3 / 2} .
$$

Using the free energy Eq. (B11), and the de Gennes expression for the coefficient of the square gradient of a polymer blend, ${ }^{3}$ we have

$$
\begin{aligned}
& \eta=\frac{1}{6} \frac{(1-\phi)^{2} V_{B}-\phi^{2} V_{A}}{\phi^{2}(1-\phi)^{2} V_{A} V_{B}}, \\
& \tau=\frac{\phi(1-\phi) \bar{V}}{(1-\phi)^{2} V_{B}-\phi^{2} V_{A}}\left(1-\frac{\chi}{\chi_{s}}\right),
\end{aligned}
$$

and

$$
\zeta=\frac{1}{36} \frac{\bar{l}}{\phi(1-\phi) l_{A} l_{B}} .
$$

The free energy barrier is then

$$
\begin{aligned}
\Delta F_{\text {nucl }} \approx & 2.23\left(\frac{\bar{l}}{l_{A} l_{B}}\right)^{3 / 2}\left(\frac{\bar{V}^{2}}{V_{A} V_{B}}\right)^{1 / 2} \\
& \times \frac{\phi(1-\phi) V_{A} V_{B}}{\left[(1-\phi)^{2} V_{B}-\phi^{2} V_{A}\right]^{2}} \bar{V}^{1 / 2}\left(1-\frac{\chi}{\chi_{s}}\right)^{3 / 2} .
\end{aligned}
$$

The $\chi$ appearing in the above equations should be interpreted as the $\chi^{e}$, in accordance with our discussions on the $\chi$-parameter issue.

${ }^{1}$ P. J. Flory, Principles of Polymer Chemistry (Cornell University Press, Ithaca, NY, 1953)

${ }^{2}$ In the literature, $\chi$ is usually defined dimensionless by using a reference volume. In our work, we avoid introducing such a reference volume; thus the $\chi$ parameter has the dimension of 1/volume.

${ }^{3}$ P.-G. de Gennes, J. Chem. Phys. 72, 4756 (1980); P. Pincus, ibid. 75, 1996 (1981).

${ }^{4}$ K. Binder, J. Chem. Phys. 79, 6387 (1983)

${ }^{5}$ K. Binder, Phys. Rev. A 29, 341 (1984).

${ }^{6}$ V. L. Ginzburg, Sov. Phys. Solid State 2, 1824 (1960); A. P. Levanyuk, Sov. Phys. JETP 36, 571 (1959).

${ }^{7}$ P.-G. de Gennes, J. Phys. Lett. (Paris) 38, L-441 (1977); see also J.-F. Joanny, J. Phys. A 11, 117 (1978).

${ }^{8}$ P.-G. de Gennes, Scaling Concepts in Polymer Physics (Cornell University Press, Ithaca, NY, 1979).

${ }^{9}$ F. S. Bates, J. Rosedale, P. Stepanik, T. P. Lodge, P. Wiltzius, G. H. Fredrickson, and R. P. Hjelm, Phys. Rev. Lett. 65, 1893 (1990).

${ }^{10}$ D. W. Hair, E. K. Hobbie, A. I. Nakatani, and C. C. Han, J. Chem. Phys. 96, 9133 (1992).

${ }^{11}$ M. Y. Belyakov and S. B. Kiselev, Physica A 190, 75 (1992).

${ }^{12}$ M. A. Anisimov, S. B. Kiselev, J. V. Sengers, and S. Tang, Physica A 188, 487 (1992).

${ }^{13}$ B. Chu, Q. Ying, K. Linliu, P. Xie, T. Gao, Y. Li, T. Nose, and M. Okada, Macromolecules 25, 7382 (1992).

${ }^{14}$ D. Schwahn, S. Janssen, and T. Springer, J. Chem. Phys. 97, 8775 (1992); D. Schwahn, G. Meir, K. Mortensen, and S. Janssen, J. Phys. II 4, 837 (1994); D. Schwahn, T. Schmackers, and K. Mortensen, Phys. Rev. E 52, R1288 (1995). 
${ }^{15}$ H. Frielinghaus, D. Schwahn, J. Dudowicz, K. F. Freed, and K. W. Foreman, J. Chem. Phys. 114, 5016 (2001).

${ }^{16}$ J. Dudowicz, M. Lifschitz, K. F. Freed, and J. F. Douglas, J. Chem. Phys. 99, 4804 (1993).

${ }^{17}$ M. Lifschitz, J. Dudowicz, and K. F. Freed, J. Chem. Phys. 100, 3957 (1994).

${ }^{18}$ A. M. Nemirovsky, M. G. Bawendi, and K. F. Freed, J. Chem. Phys. 87, 7272 (1987); J. Dudowicz and K. F. Freed, Macromolecules 24, 5076, 5096, 5112 (1991); K. F. Freed and J. Dudowicz, ibid. 29, 625 (1996).

${ }^{19}$ S. M. Wood and Z.-G. Wang, J. Chem. Phys. 116, 2289 (2002).

${ }^{20}$ A. A. Lefebvre, J. H. Lee, N. P. Balsara, and B. Hammouda, J. Chem. Phys. 116, 4777 (2002).

${ }^{21}$ B. Chu, F. J. Schoenes, and M. E. Fisher, Phys. Rev. 185, 219 (1969).

${ }^{22}$ R. J. Speedy and C. A. Angell, J. Chem. Phys. 65, 851 (1976).

${ }^{23}$ K. Binder, Ann. Phys. (N.Y.) 98, 390 (1976).

${ }^{24}$ D. W. Heermann, W. Klein, and D. Stauffer, Phys. Rev. Lett. 49, 1262 (1982).

${ }^{25}$ P. G. Debenedetti, Metastable Liquids: Concepts and Principles (Princeton University Press, Princeton, 1996).

${ }^{26}$ W. Klein and C. Unger, Phys. Rev. B 28, 445 (1983); C. Unger and W. Klein, ibid. 29, 2698 (1984)

${ }^{27}$ J. L. Lebowitz and O. Penrose, J. Math. Phys. 7, 98 (1966); O. Penrose and Lebowitz, J. Stat. Phys. 3, 211 (1971).

${ }^{28}$ Y. Saito, Prog. Theor. Phys. 59, 375 (1978).

${ }^{29}$ G. H. Fredrickson and E. Helfand, J. Chem. Phys. 87, 697 (1987).

${ }^{30}$ S. A. Brazovskii, Sov. Phys. JETP 41, 85 (1975).

${ }^{31}$ A. M. Mayes and M. O. de la Cruz, J. Chem. Phys. 95, 4670 (1991).

${ }^{32}$ E. F. David and K. S. Schweizer, J. Chem. Phys. 100, 7767 (1994); 100, 7784 (1994).

${ }^{33}$ F. S. Bates, M. Muthukumar, G. D. Wignall, and L. J. Fetters, J. Chem. Phys. 89, 535 (1988).

${ }^{34}$ C. C. Han, B. J. Bauer, J. C. Clark, V. Muroga, Y. Matsushita, M. Okada, Q. Tran-Cong, T. Chang, and I. C. Sanchez, Polymer 29, 2002 (1988)

${ }^{35}$ R. Krishnamoorti, W. W. Graessley, N. P. Balsara, and D. J. Lohse, J. Chem. Phys. 100, 3894 (1994).

${ }^{36}$ S. Janssen, D. Schwahn, K. Mortensen, and T. Springer, Macromolecules 26, 5589 (1993); B. Hammouda and B. Bauer, ibid. 28, 4505 (1995).

${ }^{37}$ T. P. Russell, T. E. Karis, Y. Gallot, and A. M. Mayes, Nature (London) 368, 729 (1994); T. E. Karis, T. P. Russell, Y. Gallot, and A. M. Mayes, Macromolecules 28, 1129 (1995).

${ }^{38}$ I. C. Sanchez and R. H. Lacombe, J. Phys. Chem. 80, 2352 (1976); R. H. Lacombe and I. C. Sanchez, ibid. 80, 2568 (1976); I. C. Sanchez and R. H. Lacombe, Macromolecules 11, 1145 (1978).

${ }^{39}$ S. K. Kumar, B. A. Veytsman, J. K. Maranas, and B. Crist, Phys. Rev. Lett. 79, 2265 (1997); M. Beiner, G. Fytas, G. Meier, and S. K. Kumar, ibid. 81, 594 (1998); S. K. Kumar, Macromolecules 33, 5285 (2000).

${ }^{40}$ J. K. Taylor, P. G. Debenedetti, W. W. Graessley, and S. K. Kumar, Macromolecules 29, 764 (1996); J. K. Taylor-Maranas, P. G. Debenedetti, W. W. Graessley, and S. K. Kumar, ibid. 30, 6943 (1997).

${ }^{41}$ K. S. Schweizer and J. G. Curro, Phys. Rev. Lett. 58, 246 (1987); J. G. Curro and K. S. Schweizer, J. Chem. Phys. 87, 1842 (1987).

${ }^{42}$ K. S. Schweizer, Macromolecules 26, 6033, 6050 (1993;).

${ }^{43}$ K. F. Freed and J. Dudowicz, Macromolecules 31, 6681 (1998).

${ }^{44}$ J. E. G. Lipson, J. Chem. Phys. 96, 1418 (1992); J. E. G. Lipson and S. S. Andrew, ibid. 96, 1426 (1992); J. E. G. Lipson and P. K. Brazhnik, ibid. 98, 8178 (1993); J. Luettmer-Strathmann and J. E. G. Lipson, Fluid Phase Equilib. 151, 649 (1998).

${ }^{45}$ P. D. Gujrati, Phys. Rev. E 54, 2723 (1996); J. Chem. Phys. 108, 5104 (1998).

${ }^{46}$ J. Melenkevitz, B. Crist, and S. K. Kumar, Macromolecules 33, 6869 (2000).
${ }^{47}$ F. S. Bates and G. H. Fredrickson, Macromolecules 27, 1065 (1994); G. H. Fredrickson, A. J. Liu, and F. S. Bates, ibid. 27, 2503 (1994).

${ }^{48}$ G. E. Garas and M. K. Kosmas, J. Chem. Phys. 103, 10790 (1995).

${ }^{49}$ M. O. de la Cruz, S. F. Edwards, and I. C. Sanchez, J. Chem. Phys. 89, 1704 (1988).

${ }^{50}$ However, with some proper modification and reinterpretation, the theory in Ref. 49 would yield results identical to ours for symmetric blends.

${ }^{51}$ C. Singh, K. S. Schweizer, and A. Yethiraj, J. Chem. Phys. 102, 2187 (1995).

${ }^{52}$ A. Yethiraj and K. S. Schweizer, J. Chem. Phys. 97, 5927 (1992); 98, 9080 (1993).

${ }^{53}$ D. Chandler, Phys. Rev. E 48, 2898 (1993).

${ }^{54}$ For a recent review of the field-theoretic methods in polymeric systems, see G. H. Fredrickson, V. Ganesan, and F. Drolet, Macromolecules 35, 16 (2002).

${ }^{55}$ D. J. Amit, Field Theory, the Renormalization Group, and Critical Phenomena (World Scientific, Singapore, 1984).

${ }^{56}$ E. Helfand and A. M. Sapse, J. Chem. Phys. 62, 1327 (1975).

${ }^{57}$ E. A. Guggenheim, Proc. R. Soc. London, Ser. A 183, 203 (1944).

${ }^{58}$ A. Sariban and K. Binder, Macromolecules 21, 711 (1988); H. P. Deutsch and K. Binder, ibid. 25, 6214 (1992).

${ }^{59}$ J. Dudowicz and K. F. Freed, Macromolecules 26, 213 (1993); J. Chem. Phys. 100, 4653 (1994).

${ }^{60}$ J. D. Londono, A. H. Narten, G. D. Wignall, K. G. Honnell, E. T. Hsieh, T. W. Johnson, and F. S. Bates, Macromolecules 27, 2864 (1994).

${ }^{61}$ W. W. Maurer, F. S. Bates, T. P. Lodge, K. Almdal, K. Mortesen, and G. H. Fredrickson, J. Chem. Phys. 108, 2989 (1998).

${ }^{62}$ R. Krishnamoorti, W. W. Graessley, N. P. Balsara, and D. J. Lohse, Macromolecules 27, 3073 (1984).

${ }^{63}$ D. J. Amit, J. Phys. C 7, 3369 (1974).

${ }^{64}$ G. Müller, D. Schwahn, and T. Springer, Phys. Rev. E 55, 7267 (1997).

${ }^{65} \mathrm{We}$ do not include the unstable part of the phase diagram in our discussion as this would require going beyond a thermodynamic treatment.

${ }^{66}$ In this discussion, we have deliberately avoided including the "=" in the inequalities involving the upper and lower critical dimensions, because subtle (often logarithmic) corrections complicate the situation in these critical dimensions.

${ }^{67}$ D. S. Corti and P. G. Debenedetti, Chem. Eng. Sci. 49, 2717 (1994); Ind. Eng. Chem. Res. 34, 3573 (1995); D. S. Corti, P. G. Debenedetti, S. Sastry, and F. H. Stillinger, Phys. Rev. E 55, 5522 (1997).

${ }^{68}$ This pseudo-spinodal is not to be confused with the same term introduced in Ref. 21.

${ }^{69}$ J. W. Cahn and J. E. Hilliard, J. Chem. Phys. 31, 688 (1959).

${ }^{70}$ A. Z. Patashinskii and B. I. Shumilo, Sov. Phys. Solid State 22, 655 (1980).

${ }^{71}$ S. B. Kiselev and I. G. Kostyukova, J. Chem. Phys. 98, 6455 (1993); S. B. Kiselev, Physica A 269, 252 (1999); S. B. Kiselev and J. F. Ely, ibid. 299, 357 (2000).

${ }^{72}$ J. S. Langer, Ann. Phys. (N.Y.) 54, 258 (1969); J. S. Langer, in Systems Far From Equilibrium, edited by L. Garrido (Springer Verlag, Heidelberg, 1980) pp. 12-47.

${ }^{73}$ N. P. Balsara, private communication.

${ }^{74}$ J. W. Cahn, Acta Metall. 9, 795 (1961); H. E. Cook, ibid. 18, 297 (1970).

${ }^{75}$ J. D. Gunton and M. C. Yalabik, Phys. Rev. B 18, 6199 (1978).

${ }^{76}$ N. P. Belsara (private communication) envisioned a similar curve based on the extrapolated divergence of the critical size of the nuclei.

${ }^{77}$ H. Tang and K. F. Freed, J. Chem. Phys. 94, 1572 (1991).

${ }^{78}$ C. Yeung, R. C. Desai, A.-C. Shi, and J. Noolandi, Phys. Rev. Lett. 72, 1834 (1994)

${ }^{79}$ K. M. Hong and J. Noolandi, Macromolecules 14, 727 (1981). 